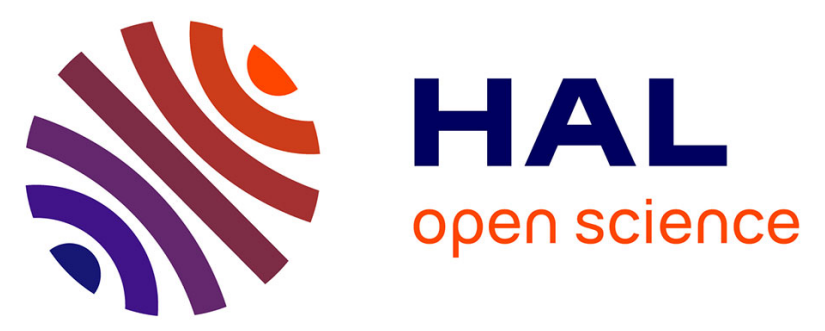

\title{
Imaging by modification: numerical reconstruction of local conductivities from corresponding power density measurements
}

Yves Capdeboscq, Jérôme Fehrenbach, Frédéric de Gournay, Otared Kavian

\section{- To cite this version:}

Yves Capdeboscq, Jérôme Fehrenbach, Frédéric de Gournay, Otared Kavian. Imaging by modification: numerical reconstruction of local conductivities from corresponding power density measurements. SIAM Journal on Imaging Sciences, 2009, 2 (4), pp.1003-1030. 10.1137/080723521 . hal-00280599v2

\section{HAL Id: hal-00280599 \\ https://hal.science/hal-00280599v2}

Submitted on 23 Jan 2009

HAL is a multi-disciplinary open access archive for the deposit and dissemination of scientific research documents, whether they are published or not. The documents may come from teaching and research institutions in France or abroad, or from public or private research centers.
L'archive ouverte pluridisciplinaire HAL, est destinée au dépôt et à la diffusion de documents scientifiques de niveau recherche, publiés ou non, émanant des établissements d'enseignement et de recherche français ou étrangers, des laboratoires publics ou privés. 


\title{
AN OPTIMAL CONTROL APPROACH TO IMAGING BY MODIFICATION
}

\author{
Y. CAPDEBOSCQ , J. FEHRENBACH , F. DE GOURNAY , AND O. KAVIAN
}

\begin{abstract}
We discuss the reconstruction of the impedance from the local power density. This study is motivated by a new imaging principle which allows to recover interior measurements of the energy density by a non invasive method. We discuss the theoretical feasibility in two dimensions, and propose numerical algorithms to recover the conductivity in two and three dimensions. The efficiency of this approach is documented by several numerical simulations.
\end{abstract}

\section{INTRODUCTION}

Let $\Omega$ be a simply connected open set in $\mathbb{R}^{d}, d=2$ or 3 , with a $C^{1}$ boundary $\partial \Omega$. Given an integer $N \geq 1$, let $\left(g_{i}\right)_{1 \leq i \leq N}$ be $N$ continuous functions in $H^{1 / 2}(\partial \Omega)$, and given $\sigma \in L^{\infty}(\Omega)$, consider $u_{i}$, solution to the following conductivity problems

$$
\operatorname{div}\left(\mathrm{e}^{\sigma} \nabla u_{i}\right)=0 \text { in } \Omega, \quad u_{i}=g_{i} \text { on } \partial \Omega, \text { for all } i \in\{1, \ldots, N\},
$$

and define $\left(S_{i j}\right)_{1 \leq i, j \leq N} \in L^{1}(\Omega)$ by

$$
S_{i j}:=\mathrm{e}^{\sigma} \nabla u_{i} \cdot \nabla u_{j} \text { a.e. in } \Omega \text {. }
$$

This work is devoted to the theoretical and numerical study of the following inverse problem: given the functions $\left(S_{i j}\right)_{1 \leq i, j \leq N}$ on $\omega \subset \subset \Omega$, and given $\sigma$ near the boundary of the domain, recover $\sigma$ inside $\omega$.

This question is motivated by a new imaging method for the determination of the conductivity inside the domain $\Omega$, which relies both on electrical impedance tomography and ultrasonic wave focusing. A focused ultrasonic wave modifies slightly the conductivity within the domain, which allows in turn to recover the energy densities $S_{i j}$. This method is described in the recent work of Ammari et al. [2]. In this paper, a first inversion algorithm is proposed to recover e ${ }^{\sigma}$ from $S_{11}, S_{22}$ and $S_{12}$, which recovers data very successfully.

Yet, many questions are left unanswered. In [2], the case of a full energy density map is considered, i.e. $\omega=\Omega$, and the ad-hoc algorithm proposed cannot be extrapolated simply when imaging smaller subdomains. This algorithm relies in a fundamental way on the existence of several measurements (at least two), and diverges in general for one measurement. Furthermore, because it is based on a perturbation approach, its stability cannot be guaranteed a priori.

This problem is studied here from a different perspective, that of optimal control. Consider the case of one current, that is, one data $S_{11}$. It is clear that the inversion can be reformulated as a minimisation problem, such as the following

$$
\text { Minimise } \mathcal{J}(\sigma):=\int_{\omega} j(E(\sigma), x) d x \text { over all } \sigma \in L^{\infty}(\Omega),
$$

where $j: \mathbb{R} \times \Omega \longrightarrow[0, \infty)$ is an appropriately chosen sufficiently smooth function and $j(s, x)=0$ iff $s=S_{11}(x)$, and

$$
E(\sigma):=\mathrm{e}^{\sigma}|\nabla u|^{2} \text { with } \operatorname{div}\left(\mathrm{e}^{\sigma} \nabla u\right)=0 \text { in } \Omega, u=g_{1} \text { on } \partial \Omega .
$$


The solving methods and numerical results presented in this paper follow this formulation, or its multi-data counterpart. To assess the quality of the inversion procedure, it is necessary to establish what can be recovered. Note that, even though the problem under consideration is related to electrical impedance tomography, we cannot rely on the theoretical results obtained for that problem: indeed we cannot assume that the Steklov-Poincaré operator, or the Dirichlet-to-Neumann map, is known, since only a limited number of voltage potentials $\left(g_{i}\right)_{1<i<N}$ are imposed on the boundary.

In fact, the nature of the inversion is quite different. For instance in the one dimensional case in which $\Omega=(0,1)$, with one data $S_{11}$ known everywhere on $(0,1)$, the conductivity is easily determined. Indeed, for some constant $C$ we have $\mathrm{e}^{\sigma} u_{x}=C$ and thus $S_{11}=\mathrm{e}^{-\sigma} C^{2}$. Also assuming for instance $g_{1}(1)-g_{1}(0)=1$, one infers that $C \int_{0}^{1} \mathrm{e}^{\sigma(x)} d x=u(1)-u(0)=1$, and $C=\int_{0}^{1} S_{11}(x) d x$ so that $C$ is determined by $S_{11}$ and finally $\sigma$ is completely determined. In contrast, using electrical impedance tomography, only the average of the conductivity over $(0,1)$ can be recovered.

Of paramount importance for this complete reconstruction is the knowledge of $S_{11}$ everywhere on $(0,1)$. In any dimension, if the data is only known on a subdomain $\omega$ strictly included in $\Omega$, one can only hope to recover the log-conductivity $\sigma$ up to an additive constant, as it is illustrated by the following example. Suppose for simplicity that $d=2$ and that the domain is a disk of radius 1 centered at the origin, and that the log-conductivity is radial, given by

$$
\sigma:= \begin{cases}\log \gamma_{0} & \text { if } r<\frac{1}{2} \\ \log \gamma_{1} & \text { if } \frac{1}{2}<r<\frac{1}{\sqrt{2}} \\ 0 & \text { if } \frac{1}{\sqrt{2}}<r<1\end{cases}
$$

In such a case, for a boundary condition of the form $g=c_{1} \cos (\theta)+s_{1} \sin (\theta)$, $\theta \in(0,2 \pi)$, the solution $u$, and, in turn, the data, can be computed explicitly by separation of variables. The solution $u$ is given in polar coordinates by $u(r, \theta)=$ $p_{1}\left(c_{1} \cos (\theta)+s_{1} \sin (\theta)\right) r$, for $r \leq 1 / 2$, where the constant $p_{1}$ is given by

$$
p_{1}=\frac{16 \gamma_{1}}{\gamma_{1}^{2}+9 \gamma_{1}+3 \gamma_{1} \gamma_{0}+3 \gamma_{0}} .
$$

We therefore see that selecting $\gamma_{0}$ and $\gamma_{1}$ such that $\gamma_{0}=\gamma_{1}\left(7-\gamma_{1}\right) / 3\left(1+\gamma_{1}\right)$ will yield the same data as that of a homogeneous medium of log-conductivity $\sigma=0$. Counter examples for polynomial boundary conditions, $g=\sum_{k=1}^{N} c_{k} \cos (k \theta)+$ $s_{k} \sin (k \theta)$ can be constructed similarly by introducing $N$ annulus of different conductivities between the disk of radius $1 / 2$ and the exterior boundary.

In Section 2, we investigate the theoretical reconstruction of the log-conductivity. First we show that, if $s_{1}=\mathrm{e}^{\sigma / 2} \nabla u_{1}$ is known, that is not only the modulus $S_{11}^{1 / 2}$ but also the direction $\theta_{1}:=\left|\nabla u_{1}\right|^{-1} \nabla u_{1}$ of the gradient $\nabla u_{1}$ is known, then the conductivity may be determined up to a multiplicative constant, under some regularity assumption (this means that the log-conductivity $\sigma$ is determined up to an additive constant). Then, we show that if two diffeomorphic measurements $s_{1}$ and $s_{2}$ are performed, yielding a set of three data $S_{11}, S_{22}$ and $S_{12}$, the direction $\theta_{1}$ can also be recovered up to a constant angle. Note that when $\omega=\Omega$, using the fact that $\sigma$ is known in a neighbourhood of $\partial \Omega$, both constants involved in the determination of the log-conductivity and that of the direction $\theta_{1}$ can be recovered. By diffeomorphic measurements we mean that the solutions $u_{1}, u_{2}$ satisfy

$$
\operatorname{det}\left(\nabla u_{1}, \nabla u_{2}\right)>0 \quad \text { or equivalently } \operatorname{det}\left(s_{1}, s_{2}\right)>0 \text { a. e. in } \omega .
$$




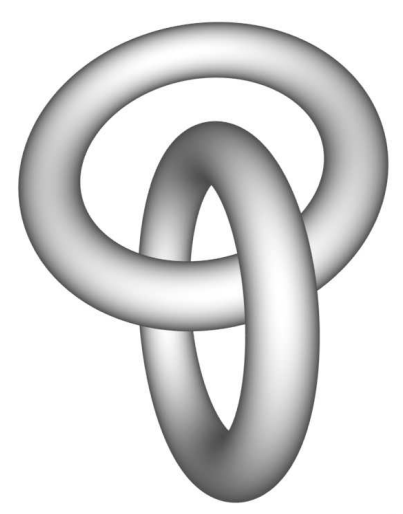

FiguRE 1. A problematic configuration for the conductivity when $d=3$ and when the conductivity is very high in the annuli compared to that of the background medium.

The difficulty is that we want to ensure that we have performed diffeomorphic measurements by a judicious choice of $g_{1}$ and $g_{2}$, independently of $\sigma$. The following theorem of Alessandrini and Nesi [1], extending the classical theorem of Radò (1926), Kneser (1926), and Choquet (1945) for harmonic maps, shows that in two dimensions, there are many such possible choices. We formulate it for the scalar case, although in [1] the result is shown for matrix valued conductivities.

Theorem 1.1 (Alessandrini \& Nesi [1]). Let $\Omega \subset \mathbb{R}^{2}$ be a bounded simply connected open set, whose boundary $\partial \Omega$ is a simple closed curve. Let $\mathbf{g}=\left(g_{1}, g_{2}\right)$ be a mapping $\partial \Omega \rightarrow \mathbb{R}^{2}$ which is a homeomorphism of $\partial \Omega$ onto a convex closed curve $\mathcal{C}$, and let $D$ denote the bounded convex domain bounded by $\mathcal{C}$. Let $\sigma \in L^{\infty}(\Omega)$, and let $U=\left(u_{1}, u_{2}\right)$ be the $\mathrm{e}^{\sigma}$-harmonic mapping whose components $u_{1}, u_{2}$ are solutions of the Dirichlet problems

$$
\begin{aligned}
\operatorname{div}\left(\mathrm{e}^{\sigma} \nabla u_{i}\right) & =0 \text { in } \Omega, \\
u_{i} & =g_{i} \text { on } \partial \Omega,
\end{aligned}
$$

with $g_{i} \in H^{1}(\Omega) \cap C(\bar{\Omega})$ and $i=1,2$. Then $U$ is a homeomorphism of $\bar{\Omega}$ onto $\bar{D}$. In particular we have either

$$
\forall \omega \subset \subset \Omega, \operatorname{det}\left(\nabla u_{1}, \nabla u_{2}\right)>0 \text { a.e. in } \omega
$$

or

$$
\forall \omega \subset \subset \Omega, \operatorname{det}\left(\nabla u_{1}, \nabla u_{2}\right)<0 \text { a.e. in } \omega
$$

As a consequence, assuming that $\Omega$ is convex and sufficiently smooth, taking measurements with, say, $g_{1}=x_{1}$ and $g_{2}=x_{2}$ (that is $\mathbf{g}=I d$ ), we are guaranteed that condition (5) holds.

Unfortunately, such a result is not true when $d=3$, even in the harmonic case as it was proved by Laugesen [9], and changes of signs in the determinant can happen at arbitrarily small scales, see Briane et al. [4]. Geometries such as the one sketched in Figure 1 provide counter-examples. These results indicate that an extension of our approach of the uniqueness problem to the three dimensional case is likely to fail. 
In Section 3, we adopt an optimisation point of view, and consider the inverse problem as described in (3). More precisely, we introduce two functionals

$$
\mathcal{J}_{1}(\sigma):=\int_{\omega}\left(\mathrm{e}^{\sigma / 2}|\nabla u|-S_{11}^{1 / 2}\right)^{2},
$$

and

$$
\mathcal{J}_{2}(\sigma):=\frac{1}{2} \sum_{i=0}^{n}\left(\int_{\omega_{i}} \mathrm{e}^{\sigma}|\nabla u|^{2} d x-\int_{\omega_{i}} S_{11} d x\right)^{2},
$$

where $\left(\omega_{i}\right)_{1 \leq i \leq n}$ is a partition of $\omega$. We compute their differentials, and show that strict convexity of such functionals cannot be guaranteed a priori. This fact is not related to the particular choice of integrand (cost functions $j$ in the language of optimal control (3)). We then discuss how a weaker lower bound on the Hessian can be used to improve minimisation procedures, and why several electric measurements are beneficial.

For the sake of concision, the gradient descent method used to minimise $\mathcal{J}_{1}$ is not detailed. In Section 4, we detail the implementation of the Gauss-Newton method used to minimise $\mathcal{J}_{2}$. For an increased numerical efficiency, the Jacobian matrix of the cost function is evaluated differently on fine and coarse meshes. This leads to a nested grid algorithm, that is, where nested subgrids appear. For three dimensional computations, the involved number of degrees of freedom prohibits the resolution of very fine scale problems. We believe that our algorithm allows to circumvent this difficulty, if a fine resolution is needed only in a small area of the domain.

Finally in Sections 5 and 6, numerical examples are presented and discussed. We first consider the two dimensional case. First we verify that perfect convergence is achieved in the most favorable case, that is, when the data and the reconstruction are done on the same mesh. We then turn to the question of stability, and perform the reconstruction on an arbitrary uniform mesh, unrelated to the one used to create the data, and introduce noise. We see that the algorithm still converges very convincingly, and reacts to the noise in a stable manner. Finally, we test a patchwork approach, that is, a simultaneous resolution on different overlapping subdomains. We then turn to the three dimensional case. The numerical issues of interpolation between different meshes are not addressed in this work, but we consider the case of noisy data. In that case the nested grids resolution method is illustrated on a half sphere - a domain resembling a breast, since breast cancer detection is an application we have in mind.

\section{On the theOREtical ReCONSTRUCtion OF THE CONDUCTIVITY IN TWO DIMENSIONS.}

In this section we show that, possibly up to two constant parameters, the conductivity can be determined from two diffeomorphic measurements. In fact, we could exhibit an explicit inversion formula from Proposition 2.1 and Proposition 2.2, using formulae (9) and (18), which would simplify the inversion to the evaluation of two real parameters, easily found from the near boundary information available for $\sigma$. We chose not to do so for two reasons. First these formulae are exclusively two dimensional, as they rely in an essential way on the fact that divergence free fields are curls, and Theorem 1.1 is used frequently. Finally, both (9) and (18) require to differentiate the measured data, and this is a well known source for instability.

Proposition 2.1. Assume $d=2$ and $\sigma \in W^{1, p}(\Omega)$ for some $p>2$ and that $\Omega$ is a smooth bounded domain. Let $u_{1}$ be the solution of (1) for $i=1$. Assume that $S_{11} \neq 0$ in $\Omega$ and furthermore that $s_{1}:=\mathrm{e}^{\sigma / 2} \nabla u_{1}$ is known almost everywhere in 
$\omega \subset \Omega$, and that

$$
\left|\nabla u_{1}\right|^{-1} \in L^{2}(\Omega) \quad \text { and } \quad\left|\nabla u_{1}\right|^{-2} \partial_{i j} u_{1} \in L^{2}(\Omega)
$$

Then $\sigma$ is known in $\omega$ up to an additive constant. Without further assumptions, the additive constant cannot always be determined.

Proof. Let $\varphi \in H_{0}^{1}(\Omega)$ be a test function. Testing (1) against $\varphi$ we obtain

$$
\int_{\Omega} \mathrm{e}^{\sigma / 2} s_{1} \cdot \nabla \varphi d x=0
$$

Recall the Sobolev embeddings $H_{0}^{1}(\Omega) \subset L^{q}(\Omega)$ for all $q<\infty$ and that $W^{1, p}(\Omega) \subset$ $C(\bar{\Omega})$ for $p>2$. Now if $\varphi \in H_{0}^{1}(\Omega)$, since $\nabla \sigma \in L^{p}(\Omega)$, upon choosing $q$ appropriately one checks that $\left(\nabla \sigma \cdot s_{1}\right) \varphi \in L^{1}(\Omega)$. This means that for any $\varphi \in H_{0}^{1}(\Omega)$ we have $\mathrm{e}^{ \pm \sigma / 2} \varphi \in H_{0}^{1}(\Omega)$ and therefore in the above identity we may replace $\varphi$ by $\mathrm{e}^{-\sigma / 2} \varphi$, which yields, for all $\varphi \in H_{0}^{1}(\Omega)$

$$
\int_{\Omega} s_{1} \cdot \nabla \varphi d x-\frac{1}{2} \int_{\Omega} \nabla \sigma \cdot s_{1} \varphi d x=0 .
$$

Now define

$$
J:=\left[\begin{array}{cc}
0 & -1 \\
1 & 0
\end{array}\right] .
$$

Then, using the fact that the vector valued function $\mathrm{e}^{-\sigma / 2} s_{1}$ is a gradient, and that we are in two dimensions, we know that $J \nabla$ is a curl and therefore for any $\varphi \in H_{0}^{1}(\Omega)$

$$
\int_{\Omega} \mathrm{e}^{-\sigma / 2} J s_{1} \cdot \nabla \varphi d x=0
$$

This time replacing $\varphi$ by $\mathrm{e}^{\sigma / 2} \varphi$ in the above identity, we obtain for all $\varphi \in H_{0}^{1}(\Omega)$

$$
\int_{\Omega} J s_{1} \cdot \nabla \varphi d x+\frac{1}{2} \int_{\Omega} \nabla \sigma \cdot J s_{1} \varphi d x=0 .
$$

Using the fact that

$$
\nabla \sigma=\left(\nabla \sigma \cdot s_{1}\right) \frac{s_{1}}{S_{11}}+\left(\nabla \sigma \cdot J s_{1}\right) \frac{J s_{1}}{S_{11}} \text { a.e. in } \Omega,
$$

for any $\Phi:=\left(\varphi_{1}, \varphi_{2}\right) \in\left(C_{c}^{1}(\Omega)\right)^{2}$ we obtain

$$
\begin{aligned}
\frac{1}{2} \int_{\Omega} \sigma \operatorname{div}(\Phi) d x & =-\frac{1}{2} \int_{\Omega} \nabla \sigma \cdot \Phi d x \\
& =-\frac{1}{2} \int_{\Omega}\left(\nabla \sigma \cdot s_{1}\right) \frac{s_{1}}{S_{11}} \cdot \Phi d x-\frac{1}{2} \int_{\Omega}\left(\nabla \sigma \cdot J s_{1}\right) \frac{J s_{1}}{S_{11}} \cdot \Phi d x \\
& =-\int_{\Omega} s_{1} \cdot \nabla\left(\frac{s_{1}}{S_{11}} \cdot \Phi\right) d x+\int_{\Omega} J s_{1} \cdot \nabla\left(\frac{J s_{1}}{S_{11}} \cdot \Phi\right) d x,
\end{aligned}
$$

where we have used (7) with $\varphi:=\Phi \cdot s_{1} / S_{11}$ and (8) with $\varphi:=\Phi \cdot J s_{1} / S_{11}$. In both cases we have $\varphi \in H_{0}^{1}(\Omega)$, since this can be readily checked, using the assumption that $\nabla \sigma \in L^{p}(\Omega),\left|\nabla u_{1}\right|^{-1} \in L^{2}(\Omega)$ and $\left|\nabla u_{1}\right|^{-2} \partial_{i j} u_{1} \in L^{2}(\Omega)$.

Now note that the right hand side of (9) depends only on the data $s_{1}$ : therefore by choosing the support of $\Phi$ in $\omega$, that is where $s_{1}$ is known, we conclude that the left hand side is known. This in turn proves that $\sigma$ is known in $\omega$ up to an additive constant.

We should observe that the assumptions on $\sigma$ and $\left|\nabla u_{1}\right|$ in the above result are not of the same nature: $\sigma$ is assumed to be sufficiently smooth, while $\nabla u_{1}$ is assumed to be away from zero. Indeed this assumption depends on the boundary data but, as we shall see below, can be realized in some circumstances. For instance one may use classical regularity results in the following way to give an example in 
which the above assumptions are satisfied. First by Morrey-Sobolev imbedding theorem we know that for some $\alpha>0$ we have $W^{1, p}(\Omega) \subset C^{0, \alpha}(\bar{\Omega})$ provided $p>2$ and $\Omega \subset \mathbb{R}^{2}$ is sufficiently smooth. Therefore, if we assume $\sigma \in W^{1, p}(\Omega)$ then $\mathrm{e}^{\sigma} \in C^{0, \alpha}(\bar{\Omega})$ and, assuming that the boundary data is sufficiently smooth, we conclude that $u \in C^{1, \alpha}(\bar{\Omega})$ (see for instance theorems 8.32-8.33 in D. Gilbarg \& N.S. Trudinger [6]). Now if we assume that $\Omega$ is convex, by the result of theorem 1.1 we conclude that $\left|\nabla u_{1}\right| \neq 0$ in $\bar{\omega}$ and since $\left|\nabla u_{1}\right|$ is Hölder continuous, we have $\left|\nabla u_{1}\right|^{-1} \in L^{\infty}(\Omega)$ and the conditions of proposition 2.1 are satisfied.

The proof of proposition 2.1 uses the fact that two orthogonal projections of the flux $s_{1}$ can be related to the conductivity. In the next proposition we show that two distinct measurements, yielding three data $S_{11}, S_{22}$ and $S_{12}$ allow to recover the direction $\theta_{1}:=\left|\nabla u_{1}\right|^{-1} \nabla u_{1}$ up to a constant angle (and in turn, the conductivity using proposition 2.1). This relies on the fact that, provided $\Omega$ is convex and smooth, if $g_{1}=x_{1}$ and $g_{2}=x_{2}$, then the solutions $u_{1}, u_{2}$ of (1) verify

$$
\operatorname{det}\left(\nabla u_{1}, \nabla u_{2}\right)>0 \text { a.e. in } \omega
$$

thanks to Theorem 1.1.

Proposition 2.2. Assume that $\Omega$ is convex and smooth, that $g_{1}=x_{1}$ and $g_{2}=x_{2}$ so that (10) holds. Assume that $S_{11}, S_{22}$ and $S_{12}$ are known in $\omega \subset \Omega$, and that $u_{1}, u_{2}$ satisfy (6). Then the direction

$$
\theta_{1}:=\frac{\nabla u_{1}}{\left|\nabla u_{1}\right|}=\frac{s_{1}}{\left|s_{1}\right|}=S_{11}^{-1 / 2} \mathrm{e}^{\sigma / 2} \nabla u_{1}
$$

is known up to a constant rotation.

Proof. First, note that $s_{1}=\mathrm{e}^{\sigma / 2} \nabla u_{1}$ and $s_{2}=\mathrm{e}^{\sigma / 2} \nabla u_{2}$ are related via two known functions of $x$. Indeed we may write, for some $\alpha(x), \beta(x)$ that $s_{2}(x)=\alpha(x) s_{1}+$ $\beta(x) J s_{1}$ and since $\operatorname{det}\left(s_{1}, s_{2}\right)=s_{1} \wedge s_{2}$ we have

$$
s_{2}=\alpha(x) s_{1}+\beta(x) J s_{1}=\frac{S_{12}}{S_{11}} s_{1}+\frac{\operatorname{det}\left(s_{1}, s_{2}\right)}{S_{11}} J s_{1} .
$$

The determinant $\operatorname{det}\left(s_{1}, s_{2}\right)$ is known, since thanks to (10) its sign is known to be positive (for instance), and therefore

$$
\operatorname{det}\left(s_{1}, s_{2}\right)=\sqrt{S_{11} S_{22}-\left(S_{12}\right)^{2}} .
$$

From equation (1), we see that for $j=1,2$ we have $\operatorname{div}\left(\mathrm{e}^{\sigma / 2} s_{j}\right)=0$. Using (11) this means that for any compactly supported test function $\varphi$,

$$
\begin{aligned}
\int_{\Omega} \mathrm{e}^{\sigma / 2} s_{1} \cdot \nabla \varphi d x & =0 \\
\int_{\Omega} \mathrm{e}^{\sigma / 2}\left(\alpha s_{1}+\beta J s_{1}\right) \cdot \nabla \varphi d x & =0 .
\end{aligned}
$$

Note that $\mathrm{e}^{-\sigma / 2} s_{j}$ is a gradient. Because $d=2, J \nabla$ is a curl and this implies

$$
\begin{aligned}
\int_{\Omega} \mathrm{e}^{-\sigma / 2} J s_{1} \cdot \nabla \varphi d x & =0, \\
\int_{\Omega} \mathrm{e}^{-\sigma / 2}\left(\alpha J s_{1}-\beta s_{1}\right) \cdot \nabla \varphi d x & =0 .
\end{aligned}
$$

For the second equation we used (11) to write $J s_{2}=\alpha J s_{1}-\beta s_{1}$. Assume momentarily that $\sigma, s_{1}$ and $s_{2}$ are smooth enough so that we can integrate by parts (13) and (15). Then using the fact that $\operatorname{div}\left(\mathrm{e}^{-\sigma / 2} J s_{1}\right)=0$, we obtain

$$
\int_{\Omega} \mathrm{e}^{\sigma / 2} \nabla \alpha \cdot s_{1} \varphi d x+\int_{\Omega} \mathrm{e}^{-\sigma / 2} \nabla\left(\mathrm{e}^{\sigma} \beta\right) \cdot J s_{1} \varphi d x=0
$$


and

$$
\int_{\Omega} \mathrm{e}^{-\sigma / 2} \nabla \alpha \cdot J s_{1} \varphi d x-\int_{\Omega} \mathrm{e}^{\sigma / 2} \nabla\left(\mathrm{e}^{-\sigma} \beta\right) \cdot s_{1} \varphi d x=0 .
$$

Introducing $\mathcal{U}=(\nabla \alpha-J \nabla \beta) \beta^{-1}$, we have proved that

$$
\int_{\Omega} \mathcal{U} \cdot s_{1} \varphi d x+\int_{\Omega} \nabla \sigma \cdot J s_{1} \varphi d x=0=\int_{\Omega} \mathcal{U} \cdot J s_{1} \varphi d x-\int_{\Omega} \nabla \sigma \cdot s_{1} \varphi d x .
$$

Integrating by parts (12), we deduce from (16) the identity,

$$
-2 \int_{\Omega} \operatorname{div}\left(s_{1}\right) \varphi d x=\int_{\Omega} \nabla \sigma \cdot s_{1} \varphi d x=\int_{\Omega} \mathcal{U} \cdot J s_{1} \varphi d x
$$

and similarly, starting from (14), thanks to (16) we obtain,

$$
-2 \int_{\Omega} \operatorname{div}\left(J s_{1}\right) \varphi d x=-\int_{\Omega} \nabla \sigma \cdot J s_{1} \varphi d x=\int_{\Omega} \mathcal{U} \cdot s_{1} \varphi d x .
$$

Finally we have proved that for any $\Phi=\left(\varphi_{1}, \varphi_{2}\right) \in C_{0}^{\infty}(\Omega)^{2}$,

$$
\begin{aligned}
\int_{\Omega} \mathcal{U} \cdot J \Phi & =\int_{\Omega}\left(\mathcal{U} \cdot s_{1}\right)\left(\frac{J \Phi \cdot s_{1}}{S_{11}}\right)+\left(\mathcal{U} \cdot J s_{1}\right)\left(\frac{J \Phi \cdot J s_{1}}{S_{11}}\right) d x \\
& =-2 \int_{\Omega}\left(\operatorname{div}\left(J s_{1}\right) \frac{J \Phi \cdot s_{1}}{S_{11}}+\operatorname{div}\left(s_{1}\right) \frac{J \Phi \cdot J s_{1}}{S_{11}}\right) d x \\
& =2 \int_{\Omega}\left(\operatorname{div}\left(J s_{1}\right) \frac{J s_{1}}{S_{11}}+\operatorname{div}\left(s_{1}\right) \frac{s_{1}}{S_{11}}\right) \cdot \Phi d x
\end{aligned}
$$

A direct computation shows that if we set $\theta_{1}:=s_{1} /\left|s_{1}\right|$, and $\theta_{1}=(\cos t, \sin t)$, we have

$$
\int_{\Omega}\left(\operatorname{div}\left(J s_{1}\right) \frac{J s_{1}}{S_{11}}+\operatorname{div}\left(s_{1}\right) \frac{s_{1}}{S_{11}}\right) \cdot \Phi d x=\int_{\Omega}\left(-J \nabla t+\nabla\left(\log S_{11}\right)\right) \cdot \Phi d x,
$$

or equivalently

$$
\int_{\Omega} J \nabla t \cdot \Phi d x=-\int_{\Omega}\left(\operatorname{div}\left(J s_{1}\right) \frac{J s_{1}}{S_{11}}+\operatorname{div}\left(s_{1}\right) \frac{s_{1}}{S_{11}}-\nabla\left(\log S_{11}\right)\right) \cdot \Phi d x .
$$

Thus we have expressed $\nabla t$ in terms of known quantities. To conclude the proof, notice that with the regularity conditions assumed about $u_{1}, u_{2}, \sigma$, the integrals involving $\mathcal{U}$ and $\nabla\left(\log S_{11}\right)$ are well defined, and therefore the function (or the angle) $t=t(x)$ is determined up to an additive constant.

\section{An optimal CONTROL APPROACH}

In Section 2, we presented Propositions 2.1 and 2.2 to document the feasibility of the inversion in an ideal case. For the practical inversion, which will be discussed in the sequel, we will adopt a very different approach. The inversion is considered as a minimisation problem, and it is performed in two or three dimensions. In Subsection 3.1, the minimisation problems are precisely stated. The direct and adjoint differentiation of the cost functions are detailed in Subsection 3.2. Such computations are relatively classical, see e.g. [5] for a electric impedance tomography equivalent. Numerical simulations are presented in Sections 5 and 6, where several boundary conditions are simultaneously considered and the effective cost functions are sum of cost functions similar to $\mathcal{J}_{1}$ or $\mathcal{J}_{2}$. Subsection 3.3 is devoted to the study of the convexity properties of the cost functions. We consider the general form (3) of the minimisation problem, and discuss the convexity properties of the minimisation of

$$
\mathcal{J}(\sigma)=\int_{\Omega} j(E(\sigma), x) d x
$$


As in (3), we assume that $j: \mathbb{R} \times \Omega \rightarrow L^{1}(\Omega)$ is a Caratheodory function which is $C^{2}$ with respect to its first variable, non-negative, and such that $j(s, x)=0$ if and only if $s=\mathrm{e}^{\sigma^{\star}(x)}\left|\nabla u_{\star}(x)\right|^{2}$, where $\mathrm{e}^{\sigma^{\star}}$ is the true conductivity and $u_{\star}$ is the associated electric potential. Strictly convex functionals are known to be favourable in optimisation problems, as they imply uniqueness of the solution and convergence of descent algorithms. We show that the minimisation is not fault free, namely, around the global minimiser, the Hessian of $\mathcal{J}$ is not positive definite.

3.1. The minimisation problems. We assume that the true log-conductivity $\sigma^{\star}$ is known in a layer of positive thickness close to the boundary $\partial \Omega$. More precisely: we assume that $\sigma^{\star}$ is known in $\Omega \backslash \Omega^{\prime}$, where $\Omega^{\prime}$ is a subdomain such that $\operatorname{dist}\left(\Omega^{\prime}, \partial \Omega\right)>0$. Let $g \in H^{1 / 2}(\partial \Omega)$. The set of admissible log-conductivities is

$$
L_{\mathrm{ad}}^{\infty}(\Omega)=\left\{\sigma \in L^{\infty}(\Omega)|\sigma|_{\Omega \backslash \Omega^{\prime}}=\sigma^{\star}\right\},
$$

and its tangent space at any point is the subspace denoted by

$$
L_{0}^{\infty}(\Omega)=\left\{\delta \in L^{\infty}(\Omega)|\delta|_{\Omega \backslash \Omega^{\prime}}=0\right\} .
$$

If $\sigma \in L_{\mathrm{ad}}^{\infty}(\Omega)$, we denote by $u(\sigma) \in H^{1}$ the solution $u$ of

$$
\begin{cases}\nabla \cdot\left(\mathrm{e}^{\sigma} \nabla u\right)=0 & \Omega \\ u=g & \partial \Omega .\end{cases}
$$

We describe two optimisation methods to recover the conductivity from energy density measurements. The heuristic idea is to find $\sigma \in L_{\mathrm{ad}}^{\infty}(\Omega)$ such that

$$
E(\sigma):=\mathrm{e}^{\sigma}|\nabla u|^{2}
$$

approaches $S=E\left(\sigma^{\star}\right)$. For the sake of concision we write sometimes $E(\sigma)$ instead of $E(\sigma, x)=\mathrm{e}^{\sigma(x)}|\nabla u(x)|^{2}$.

The first method uses local measurements of $S$ on the subdomain $\omega$ : it consists in minimising the following cost function

$$
\mathcal{J}_{1}(\sigma)=\int_{\omega}\left[\sqrt{E(\sigma)}-\sqrt{E\left(\sigma^{\star}\right)}\right]^{2} d x
$$

This corresponds to the general problem (19) with $j(s, x)=\left(\sqrt{s}-\sqrt{E\left(\sigma^{\star}, x\right)}\right)^{2}$ for $x \in \omega$ and $j(s, x)=0$ otherwise.

The second method is a nested grid method. The domain $\Omega^{\prime}$ is partitioned in subdomains $\left(\omega_{i}\right)_{1 \leq i \leq n}$. Let $\omega_{0}=\Omega \backslash \Omega^{\prime}$. We assume that the total power in each $\omega_{i}$ $(i=0 \ldots n)$ is known, this quantity is $\int_{\omega_{i}} E\left(\sigma^{\star}\right)$. The cost function to be minimised is:

$$
\mathcal{J}_{2}(\sigma)=\frac{1}{2} \sum_{i=0}^{n}\left(\int_{\omega_{i}} E(\sigma) d x-\int_{\omega_{i}} E\left(\sigma^{\star}\right) d x\right)^{2}
$$

where we restrict to conductivities that are constant on each $\omega_{i}$. Note that if a finite element method with piecewise constant conductivities on a mesh $\left(T_{i}\right)_{1 \leq i \leq M}$ is used for the discretisation of (19) with $j(s, x)=\left(s-E\left(\sigma^{\star}, x\right)\right)^{2}$, then the minimisation problem of (19) and the minimisation of $\mathcal{J}_{2}$ are equivalent when $n=M$ and for all $i \in\{1, \ldots, M\}, \omega_{i}=T_{i}$ if piecewise linear $\left(P_{1}\right)$ finite elements are used.

To minimise these functionals, we shall use either a steepest descent algorithm with $\mathcal{J}_{1}$, or a Gauss-Newton algorithm with $\mathcal{J}_{2}$. Both approaches require differentiations. 
3.2. Differentiation of $\mathcal{J}_{1}$ and $\mathcal{J}_{2}$. It is well known that the mapping $\sigma \mapsto u(\sigma)$ defined on $L_{\text {ad }}^{\infty}(\Omega) \rightarrow H^{1}(\Omega)$ is $C^{\infty}$ (in fact analytic), and that if $\delta \in L_{0}^{\infty}(\Omega)$, the differential of $u$ in the $\delta$ direction is $\mathrm{d} u . \delta=v$ where $v \in H_{0}^{1}(\Omega)$ is the solution of the variational problem

(23) $v \in H_{0}^{1}(\Omega), \quad \forall \varphi \in H_{0}^{1}(\Omega), \quad \int_{\Omega} \mathrm{e}^{\sigma} \nabla v \cdot \nabla \varphi d x=-\int_{\Omega} \delta \mathrm{e}^{\sigma} \nabla u \cdot \nabla \varphi d x$.

Consider the mapping

$$
\begin{aligned}
E: L_{\mathrm{ad}}^{\infty}(\Omega) & \longrightarrow L^{1}(\Omega) \\
\sigma & \longmapsto \mathrm{e}^{\sigma}|\nabla u(\sigma)|^{2} .
\end{aligned}
$$

The mapping $E$ is clearly differentiable, and its derivative in the $\delta$ direction is given by:

$$
\mathrm{d} E . \delta=\delta \mathrm{e}^{\sigma}|\nabla u|^{2}+2 \mathrm{e}^{\sigma} \nabla u \cdot \nabla v,
$$

where $v=\mathrm{d} u . \delta$ is the unique solution of $(23)$.

Proposition 3.1. The operator $\mathrm{d} E: L_{0}^{\infty}(\Omega) \rightarrow L^{1}(\Omega)$ has the following symmetry property: for all $\delta, \eta \in L_{0}^{\infty}(\Omega)$, denoting by $\langle\cdot, \cdot\rangle$ the duality between $L^{1}(\Omega)$ and $L^{\infty}(\Omega)$ we have

$$
\langle\mathrm{d} E . \delta, \eta\rangle=\langle\mathrm{d} E . \eta, \delta\rangle .
$$

Proof. If $\eta \in L_{0}^{\infty}(\Omega)$, then

$$
\mathrm{d} E . \eta=\eta \mathrm{e}^{\sigma}|\nabla u(\sigma)|^{2}+2 \mathrm{e}^{\sigma} \nabla u(\sigma) . \nabla w,
$$

where $w$ solves

$$
\begin{cases}\nabla \cdot\left(\mathrm{e}^{\sigma} \nabla w\right)=-\nabla \cdot\left(\eta \mathrm{e}^{\sigma} \nabla u(\sigma)\right) & \Omega \\ w=0 & \partial \Omega .\end{cases}
$$

We can write

$$
\langle\mathrm{d} E . \delta, \eta\rangle_{L^{1}, L^{\infty}(\Omega)}=\int_{\Omega} \eta \delta \mathrm{e}^{\sigma}|\nabla u(\sigma)|^{2}+2 \eta \mathrm{e}^{\sigma} \nabla u(\sigma) \cdot \nabla v .
$$

The second term in this integral is evaluated by:

$$
\int_{\Omega} \eta \mathrm{e}^{\sigma} \nabla u(\sigma) \cdot \nabla v=-\int_{\Omega} v \nabla \cdot\left(\eta \mathrm{e}^{\sigma} \nabla u(\sigma)\right)=-\int_{\Omega} \mathrm{e}^{\sigma} \nabla v \cdot \nabla w .
$$

As a result,

$$
\langle\mathrm{d} E . \delta, \eta\rangle_{L^{1}, L^{\infty}(\Omega)}=\int_{\Omega} \eta \delta \mathrm{e}^{\sigma}|\nabla u(\sigma)|^{2}-2 \int_{\Omega} \mathrm{e}^{\sigma} \nabla v \cdot \nabla w .
$$

This quantity being symmetric in $\delta$ and $\eta$, it follows that

$$
\langle\mathrm{d} E . \delta, \eta\rangle_{L^{1}, L^{\infty}(\Omega)}=\langle\mathrm{d} E . \eta, \delta\rangle_{L^{1}, L_{0}^{\infty}(\Omega)}
$$

and the result is proved.

As a consequence of (23), if we write

$$
\mathcal{J}_{2}(\sigma)=\frac{1}{2}\|F(\sigma)\|^{2}
$$

where

(26) $\quad F(\sigma)=\left(f_{i}(\sigma)\right)_{0 \leq i \leq n} \in \mathbb{R}^{n+1} \quad$ with $\quad f_{i}(\sigma)=\int_{\omega_{i}} E(\sigma)-\int_{\omega_{i}} E\left(\sigma^{\star}\right)$,

the mapping $F: L_{\text {ad }}^{\infty}(\Omega) \rightarrow \mathbb{R}^{n+1}$ defined in (26) is differentiable, and if $\sigma \in L_{\text {ad }}^{\infty}(\Omega)$ and $\delta \in L_{0}^{\infty}(\Omega)$ :

$$
\mathrm{d} F . \delta=\left(\mathrm{d} f_{0} . \delta, \mathrm{d} f_{1} . \delta, \ldots, \mathrm{d} f_{n} . \delta\right)
$$


with

$$
\mathrm{d} f_{i} . \delta=\int_{\omega_{i}}\left(\delta+2 \frac{\nabla u(\sigma) \cdot \nabla v}{|\nabla u(\sigma)|^{2}}\right) \mathrm{e}^{\sigma}|\nabla u(\sigma)|^{2} d x
$$

where $v$ solves (23).

Let us now study the adjoint differentiation of $\mathcal{J}_{1}$, and $\mathcal{J}_{2}$.

Proposition 3.2. Assume that $\omega \subset \Omega^{\prime}$. Let $\chi$ be the characteristic function of $\omega$. Define an error function

$$
\varepsilon(\sigma):=\chi \mathrm{e}^{\sigma}\left(\sqrt{\frac{E\left(\sigma^{\star}\right)}{E(\sigma)}}-1\right),
$$

and let $p$ be the adjoint state solution of the variational problem

$$
p \in H_{0}^{1}(\Omega), \quad \forall \varphi \in H_{0}^{1}(\Omega) \quad \int_{\Omega} \mathrm{e}^{\sigma} \nabla p \cdot \nabla \varphi d x=\int_{\Omega} \varepsilon \nabla u \cdot \nabla \varphi d x .
$$

The functional $\mathcal{J}_{1}$ is differentiable with respect to $\sigma$ and its derivative is given by

$$
\mathrm{d} \mathcal{J}_{1} \cdot \delta=\int_{\Omega} \delta\left(-\varepsilon+2 \frac{\nabla u \cdot \nabla p}{|\nabla u|^{2}}\right) \mathrm{e}^{\sigma}|\nabla u|^{2} d x .
$$

Proof. Note that $p \in H_{0}^{1}(\Omega)$ is solution to the elliptic adjoint problem $\operatorname{div}\left(\mathrm{e}^{\sigma} \nabla p\right)=$ $\operatorname{div}(\varepsilon(\sigma) \nabla u)$. We have

$$
\mathcal{J}_{1}(\sigma)=\int_{\Omega} \chi\left(\sqrt{E(\sigma)}-\sqrt{E\left(\sigma^{\star}\right)}\right)^{2} d x
$$

and so the derivative of $\mathcal{J}_{1}$ is:

(29) $\mathrm{d} \mathcal{J}_{1} . \delta=\int_{\Omega} \chi\left(\sqrt{E(\sigma)}-\sqrt{E\left(\sigma^{\star}\right)}\right)\left(\delta \mathrm{e}^{\sigma / 2}|\nabla u|+2 \mathrm{e}^{\sigma / 2} \frac{\nabla v \cdot \nabla u}{|\nabla u|}\right) d x$,

where $v=\mathrm{d} u . \delta$ is defined in equation (23). In order to write the derivative $\mathrm{d} \mathcal{J}_{1}$ in terms of $\delta$ only - and not in terms of $\delta$ and $v$ - an adjoint problem is used. Namely let $p \in H_{0}^{1}$ be the solution of:

$$
\begin{aligned}
& p \in H_{0}^{1}(\Omega), \quad \forall \varphi \in H_{0}^{1}(\Omega) \\
& \int_{\Omega} \mathrm{e}^{\sigma} \nabla p \cdot \nabla \varphi d x=-\int_{\Omega} \chi\left(\sqrt{E(\sigma)}-\sqrt{E\left(\sigma^{\star}\right)}\right) \mathrm{e}^{\sigma / 2} \frac{\nabla u \cdot \nabla \varphi}{|\nabla u|} d x \\
&=\int_{\Omega} \varepsilon \nabla u \cdot \nabla \varphi d x
\end{aligned}
$$

Then, choosing $\varphi:=v$ in equation (31), it follows from equation (23) with $\varphi=p$ that:

$$
\mathrm{d} \mathcal{J}_{1} . \delta=\int_{\Omega}\left(\chi \mathrm{e}^{\sigma}|\nabla u|^{2}\left(1-\sqrt{E\left(\sigma^{\star}\right) / E(\sigma)}\right)+2 \mathrm{e}^{\sigma} \nabla u \nabla p\right) \delta d x,
$$

which is $(28)$.

Proposition 3.3. Let $\sigma \in L_{\mathrm{ad}}^{\infty}(\Omega)$ and $Z=\left(z_{0}, z_{1}, \ldots, z_{n}\right) \in \mathbb{R}^{n+1}$. Then

$$
\mathrm{d} F^{T} \cdot Z=z|\nabla u|^{2}+2 \nabla u \cdot \nabla p,
$$

where $z$ is the piecewise constant function equal to $z_{i}$ in $\omega_{i}(i=0 \ldots, n)$ and $p$ solves

$$
\begin{cases}\nabla \cdot\left(\mathrm{e}^{\sigma} \nabla p\right)=\nabla \cdot\left(z \mathrm{e}^{\sigma} \nabla u\right) & \Omega \\ p=0 & \partial \Omega .\end{cases}
$$


This for instance allows us to compute the gradient of the cost-function $\mathcal{J}_{2}$. Indeed, since $\mathcal{J}_{2}(\sigma)=\frac{1}{2}\|F\|^{2}$ we have

$$
\nabla \mathcal{J}_{2}=\mathrm{d} F^{T} \cdot F
$$

and so it suffices to apply (31) with $Z=F$.

Proof of Proposition 3.3. If $\delta \in L_{0}^{\infty}(\Omega)$, then

$$
\left\langle\mathrm{d} F^{T} . Z, \delta\right\rangle_{L^{1}, L^{\infty}(\Omega)}=\langle Z, \mathrm{~d} F . \delta\rangle_{\mathbb{R}^{n+1}}=\sum_{i=0}^{n} z_{i} \int_{\omega_{i}} \delta \mathrm{e}^{\sigma}|\nabla u|^{2}+2 \mathrm{e}^{\sigma} \nabla u \cdot \nabla v d x .
$$

Using the definition of $z$ this can be written

$$
\left\langle\mathrm{d} F^{T} . Z, \delta\right\rangle_{L^{1}, L_{0}^{\infty}(\Omega)}=\int_{\Omega} z \delta \mathrm{e}^{\sigma}|\nabla u(\sigma)|^{2}+2 z \mathrm{e}^{\sigma} \nabla u(\sigma) \cdot \nabla v d x .
$$

Let us evaluate the second term in the right-hand side. Choosing $v$ as a test function in the adjoint equation satisfied by $p$, and choosing $p$ as a test function in the equation satisfied by $v$, we have

$$
\int_{\Omega} z \mathrm{e}^{\sigma} \nabla u(\sigma) \cdot \nabla v d x=\int_{\Omega} \mathrm{e}^{\sigma} \nabla p \cdot \nabla v d x=\int_{\Omega} \delta \mathrm{e}^{\sigma} \nabla p \cdot \nabla u d x .
$$

Hence for every $\delta \in L_{0}^{\infty}(\Omega)$,

$$
\left\langle\mathrm{d} F^{T} \cdot Z, \delta\right\rangle_{L^{1}, L^{\infty}(\Omega)}=\int_{\Omega}\left(z \mathrm{e}^{\sigma}|\nabla u|^{2}+2 \mathrm{e}^{\sigma} \nabla u \cdot \nabla p\right) \delta d x .
$$

3.3. Local minimisers and degenerate convexity. We make the following simple observation.

Proposition 3.4. Assume that $\sigma$ is such that $E(\sigma)=E\left(\sigma^{\star}\right)$. Then

$$
\left|D^{2} \mathcal{J}(\sigma) \cdot[\delta, \delta]\right| \leq 2\left\|\frac{d^{2} j}{d s^{2}}\left(E\left(\sigma^{\star}\right), x\right) E\left(\sigma^{\star}\right)\right\|_{\infty} D^{2} \mathcal{J}_{1}(\sigma) \cdot[\delta, \delta],
$$

and whenever $\left|\frac{d^{2} j}{d s^{2}}\left(E\left(\sigma^{\star}\right), x\right)\right|^{-1}$ is integrable we have

$$
\left(\int_{\Omega}\left|\frac{d^{2} j}{d s^{2}}\left(E\left(\sigma^{\star}\right), x\right)\right|^{-1} d x\right)^{-1}\left(\int_{\Omega} E \delta d x\right)^{2} \leq D^{2} \mathcal{J} \cdot[\delta, \delta] .
$$

When $\mathcal{J}=\mathcal{J}_{1}$, we have

$$
\frac{1}{2}\left(\int_{\Omega} E\left(\sigma^{\star}\right) d x\right)^{-1}\left(\int_{\Omega} E\left(\sigma^{\star}\right) \delta d x\right)^{2} \leq D^{2} \mathcal{J}_{1}(\sigma) \cdot[\delta, \delta] \leq \frac{1}{2} \int_{\Omega} E\left(\sigma^{\star}\right) \delta^{2} d x .
$$

Note that Proposition 3.4 illustrates the fact that it is sufficient to study the case $\mathcal{J}=\mathcal{J}_{1}$ to prove that degeneracy in the convexity is general. In particular, inequality (33) shows that the Hessian of $\mathcal{J}$ cancels simultaneously as that of $\mathcal{J}_{1}$, if the energy density is smooth.

The lower bound (34) does not prove strict convexity. However, it provides a rule-of-thumb on how to mend steepest descent algorithm resolutions that tend to stall close to the minimiser. Since $E\left(\sigma^{\star}\right)$ is strictly positive, the lower bound will not vanish if $\delta \geq 0$, or $\delta \leq 0$ everywhere. Therefore, if $\delta^{\star}$ is the increment given by the algorithm, choosing to alternatively apply either $\max \left(\delta^{\star}, 0\right)$ or $\min \left(\delta^{\star}, 0\right)$ will avoid a degenerate behaviour. 
Proof of Proposition 3.4. Performing the same computation as in the proof of Proposition 3.2 , we have for $\sigma \in L_{\mathrm{ad}}^{\infty}(\Omega)$ and $\delta \in L_{0}^{\infty}(\Omega)$,

$$
D \mathcal{J}(\sigma) \cdot \delta=\int_{\Omega} \frac{\partial j}{\partial s}(E(\sigma), x) E(\sigma)\left[\delta+2 \frac{\nabla v \cdot \nabla u}{|\nabla u|^{2}}\right] d x .
$$

Another differentiation shows that the second derivative is given by

$$
\begin{aligned}
D^{2} \mathcal{J}(\sigma) \cdot[\delta, \delta] & =\int_{\Omega} \frac{\partial^{2} j}{\partial s^{2}}(E(\sigma), x)\left[E(\sigma)\left(\delta+2 \frac{\nabla v \cdot \nabla u}{|\nabla u|^{2}}\right)\right]^{2} d x \\
& +\int_{\Omega} \frac{\partial j}{\partial s}(E(\sigma), x) E(\sigma)\left[\delta^{2}+4 \delta \frac{\nabla v \cdot \nabla u}{|\nabla u|^{2}}+2 \frac{\nabla w \cdot \nabla u}{|\nabla u|^{2}}\right] d x
\end{aligned}
$$

where $w \in H_{0}^{1}(\Omega)$ is the second derivative of $u(\sigma)$ in the direction $\delta$. When $\frac{\partial j}{\partial s}(E(\sigma), x) \equiv 0$, that is at a critical point of $\mathcal{J}$, this simplifies in

$$
D^{2} \mathcal{J}(\sigma) \cdot[\delta, \delta]=\int_{\Omega} \frac{d^{2} j}{d s^{2}}(E(\sigma), x)\left[E(\sigma)\left(\delta+2 \frac{\nabla v \cdot \nabla u}{|\nabla u|^{2}}\right)\right]^{2} d x
$$

Note that at the global minimiser, that is when $E(\sigma)=E\left(\sigma^{\star}\right)$ a.e. in $\omega$, by construction identity (35) holds and $\frac{\partial^{2} j}{\partial s^{2}}(E(\sigma), x) \geq 0$. Therefore,

$$
\begin{aligned}
\left|D^{2} \mathcal{J}(\sigma) \cdot[\delta, \delta]\right| & \leq\left\|\frac{d^{2} j}{d s^{2}}\left(E\left(\sigma^{\star}\right), x\right) E\left(\sigma^{\star}\right)\right\|_{\infty} \int_{\omega} E(\sigma)\left[\delta+2 \frac{\nabla v \cdot \nabla u}{|\nabla u|^{2}}\right]^{2} d x \\
& \leq 2\left\|\frac{\partial^{2} j}{\partial s^{2}}\left(E\left(\sigma^{\star}\right), x\right) E\left(\sigma^{\star}\right)\right\|_{\infty} D^{2} \mathcal{J}_{1}(\sigma) \cdot[\delta, \delta],
\end{aligned}
$$

upon applying formula (35) to

$$
j(s, x)=\left(s^{1 / 2}-E\left(\sigma^{\star}, x\right)^{1 / 2}\right)^{2} .
$$

This proves the first part of the proposition. Let us now turn to $\mathcal{J}_{1}$. Expanding $D^{2} \mathcal{J}_{1}(\sigma) \cdot[\delta, \delta]$, we find

$$
\begin{aligned}
D^{2} \mathcal{J}_{1}(\sigma) \cdot[\delta, \delta] & =\frac{1}{2} \int_{\Omega} E(\sigma) \delta^{2} d x+2 \int_{\Omega} E(\sigma)\left[\frac{\nabla v \cdot \nabla u}{|\nabla u|^{2}}\right]^{2} d x \\
& +2 \int_{\Omega} \delta \nabla u \cdot \nabla v d x \\
& =\frac{1}{2} \int_{\Omega} E(\sigma) \delta^{2} d x+2 \int_{\Omega} \frac{\mathrm{e}^{\sigma}}{|\nabla u|^{2}}\left([\nabla v \cdot \nabla u]^{2}-|\nabla v|^{2}|\nabla u|^{2}\right) d x
\end{aligned}
$$

where we have used (23) with $\varphi=v$. Since, by Cauchy-Schwarz inequality, the second term of the right-hand-side is non positive, we have shown that

$$
D^{2} \mathcal{J}_{1}(\sigma) \cdot \delta \cdot \delta \leq \frac{1}{2} \int_{\Omega} E(\sigma) \delta^{2} d x
$$

On the other hand, if we choose $v$ as a test function in (20) and integrate by parts, we obtain

$$
\int_{\Omega} E(\sigma) \frac{\nabla v \cdot \nabla u}{|\nabla u|^{2}} d x=0
$$


As a consequence,

$$
\begin{aligned}
\left(\int_{\Omega} E(\sigma) \delta d x\right)^{2} & =\left(\int_{\Omega} E(\sigma)\left[\delta+2 \frac{\nabla v \cdot \nabla u}{|\nabla u|^{2}}\right] d x\right)^{2} \\
& \leq D^{2} \mathcal{J} \cdot[\delta, \delta]\left(2 \int_{\Omega} E(\sigma) d x\right)
\end{aligned}
$$

using again Cauchy-Schwarz inequality.

The next proposition shows that the Hessian $D^{2} \mathcal{J}$ is not always positive definite. We provide a counter example in the simplest case, that is, for the Laplacian.

Proposition 3.5. Let $\Omega:=(0, \pi)^{2}$ and assume that $\sigma \equiv 0$, while $g=x_{1}$, and $\partial j / \partial s(E(0), x) \equiv 0$. Then there exist directions $\delta$ such that

$$
\|\delta\|_{L^{2}(\Omega)}=1 \quad \text { and } \quad D^{2} \mathcal{J}(0)[\delta, \delta]=0 .
$$

Proof. Since $\sigma=0$, we have $u:=u_{1}=x_{1}$. The corrector $v \in H_{0}^{1}$ given by (23) satisfies

$$
\Delta v=-\nabla_{x} \delta
$$

Choose

$$
\begin{aligned}
\delta(x, y) & =\sum_{n=1}^{\infty} 2 d_{n} \cos (n x) \sin (n y) \\
& =\sum_{n=1}^{\infty} d_{n} \sin (n(x+y))+\sum_{n=1}^{\infty} d_{n} \sin (n(y-x))
\end{aligned}
$$

with

An explicit computation gives

$$
\frac{\pi}{2} \sum_{n=1}^{\infty} d_{n}^{2}=1
$$

$$
v(x, y)=-\sum_{n=1}^{\infty} \frac{1}{2 n} d_{n} \sin (n x) \sin (n y)
$$

and

$$
\delta+2 \frac{\nabla u \cdot \nabla v}{|\nabla u|^{2}}=0 \text { in } \Omega .
$$

Therefore, since from formula (35) we have

$$
D^{2} \mathcal{J}(0)[\delta, \delta]=\frac{1}{2} \int_{\Omega} \frac{d^{2} j}{d s^{2}}(E(0), x)\left[E(0)\left(\delta+2 \frac{\nabla u \cdot \nabla v}{|\nabla u|^{2}}\right)\right]^{2} d x
$$

the proof is complete.

Remark: In the proof of Proposition 3.5, we selected an ad-hoc family of perturbations, to cancel the Hessian. Had we started with a perturbation of the general form

$$
\delta=\sum_{n \geq 0} \sum_{p>0} d_{n, p} \cos (n x) \sin (p y)
$$

we would have obtained

$$
\left(\delta+2 \frac{\nabla u \cdot \nabla v}{|\nabla u|^{2}}\right)^{2}=\sum_{p>0} d_{0, p}^{2} \sin ^{2}(p y)+\sum_{n>0, p>0} d_{n, p}^{2}\left(\frac{n^{2}-p^{2}}{n^{2}+p^{2}}\right)^{2} \cos ^{2}(n x) \sin ^{2}(p y) .
$$

The first sum on the right-hand-side corresponds to the Hessian along the $\nabla u /|\nabla u|=$ $(1,0)$ direction. These terms are not weighted by any attenuation factor. Therefore 
the increment in a steepest descent algorithm is the most important in that direction. This, we think, is an explanation for the speed-up observed in computations, when multiple currents are used, simultaneously or alternatively: the optimisation becomes efficient in multiple directions.

\section{Minimisation of the COSt Function $\mathcal{J}_{2}$}

This section is dedicated to the presentation of the minimisation algorithm for $\mathcal{J}_{2}$. We do not detail the gradient descent — or steepest descent — algorithm used for $\mathcal{J}_{1}$. It is a steepest descent algorithm with adaptive step that was implemented using FreeFem++ [8]. Several boundary conditions are used to estimate the conductivity: $g_{1}, \ldots, g_{N}$. The cost function is the sum of the cost-functions associated to these boundary conditions: $\mathcal{J}_{1}=\sum_{1 \leq k \leq N} \mathcal{J}_{1}^{k}$. Note that the positivity constraint for the conductivity is automatically satisfied by the choice of a log-conductivity $\left(e^{\sigma}>0\right)$.

The optimisation procedures used to minimise the cost function $\mathcal{J}_{2}$ are detailed. Although we always use Gauss-Newton method, a different approach is followed for fine or coarse computations, which yields in turn a nested grid algorithm, presented in Section 4.2 .

4.1. Gauss-Newton optimisation. We make use of several boundary conditions to estimate the conductivity: $g_{1}, \ldots, g_{N}$. The cost function is the sum of the costfunctions associated to these boundary conditions: $\mathcal{J}_{2}=\sum_{1 \leq k \leq N} \mathcal{J}_{2}^{k}$.

The domain $\Omega$ is divided in subdomains $\left(\omega_{i}\right)_{0 \leq i \leq n}$. The true value of the conductivity in $\omega_{0}$ is assumed to be known. We consider $F: \mathbb{R}^{n} \rightarrow\left(\mathbb{R}^{n}\right)^{N}, \sigma \mapsto$ $\left(F_{1}(\sigma), \ldots, F_{N}(\sigma)\right)$, where $F_{k}$ is associated to the boundary condition $g_{k}$ and is defined as in equation (26). The map $F$ is $C^{1}$ and $\mathrm{d} F=\left(\mathrm{d} F_{1}, \ldots, \mathrm{d} F_{N}\right)$.

The Gauss-Newton Method is an iterative descent method designed to minimise a cost function of the form

$$
\mathcal{J}_{2}(\sigma)=\frac{1}{2}\|F(\sigma)\|^{2}=\sum_{k=1}^{N}\left\|F_{k}(\sigma)\right\|^{2} .
$$

An initial guess $\sigma^{0}$ is provided, and the $m$-th iteration reads $\sigma^{m+1}:=\sigma^{m}+\delta^{m}$, where the descent direction $\delta^{m}$ solves

$$
\mathrm{d} F^{T} \cdot \mathrm{d} F \cdot \delta^{m}=-\mathrm{d} F^{T} . F .
$$

If the number $n$ of unknowns is small (typically $n$ smaller than 20) the Jacobian matrices $\mathrm{d} F_{k}$ are computed column-wise by solving $n$ conductivity problems using formula (27). The equation (37) is then assembled and solved easily using any linear solver since $\mathrm{d} F^{T} \cdot \mathrm{d} F=\sum_{k=1}^{N} \mathrm{~d} F_{k}^{T} \cdot \mathrm{d} F_{k}$ and $\mathrm{d} F^{T} \cdot F=\sum_{k=1}^{N} \mathrm{~d} F_{k}^{T} \cdot F_{k}$.

The step $\delta^{m}$ obtained is used as is, without further modification. If the number of unknowns $n$ is large (for a fine spatial resolution there can be one unknown value of the conductivity in each convex on the finite element mesh) the computation of the full matrix $\mathrm{d} F$ is time and memory consuming. We use an iterative method, namely the conjugate gradient, to solve equation (37) without assembling $\mathrm{d} F$. This requires merely the knowledge of the right-hand side $\mathrm{d} F^{T} . F$ and a procedure that gives the product of the matrix $\mathrm{d} F^{T} \cdot \mathrm{d} F$ by a given vector. These are provided by the direct and adjoint differentiations described in paragraph 3.2.

More precisely: $F\left(\sigma^{m}\right)$ is computed by solving $N$ conductivity problems; $\mathrm{d} F^{T} . F$ is computed by adjoint differentiation $(N$ adjoint problems of conductivity to be solved); and if $\xi$ is given, $\mathrm{d} F^{T} \cdot \mathrm{d} F \xi=\mathrm{d} F^{T} .(\mathrm{d} F . \xi)$ is computed in two steps, solving $d$ direct then $d$ adjoint conductivity problems.

We implemented the algorithms that are sketched below: 
Algorithm 1: multiple sub-domains conductivity estimation (full Jacobian)

input: the values of the boundary currents $g_{1}, \ldots, g_{N}$ and the associated measurements $E_{1}\left(\sigma^{\star}\right), \ldots, E_{N}\left(\sigma^{\star}\right)$, initial guess $\sigma^{0}$ (such that $\sigma^{0}=\sigma^{\star}$ in $\Omega \backslash \omega$ )

1.- set $m:=0$,

2.- compute the potentials $u_{1}, \ldots, u_{N}$ predicted with the log-conductivity $\sigma^{m}$,

3.- compute $F_{k}=\left(f_{k, 1}, f_{k, 2}, \ldots, f_{k, n}\right)$ where $f_{k, i}=\int_{\omega_{i}} \mathrm{e}^{\sigma^{m}}\left|\nabla u_{k}\right|^{2}-\int_{\omega_{i}} E_{k}\left(\sigma^{\star}\right)$, for $k=1 \ldots, N$,

4.- compute $\mathrm{d} F_{k}, k=1 \ldots, N$ column-wise using equation (27),

5.- solve for $\delta$ the equation $\left(\sum_{1 \leq k \leq N} \mathrm{~d} F_{k}^{T} \cdot \mathrm{d} F_{k}\right) \delta=-\sum_{1 \leq k \leq N} \mathrm{~d} F_{k}^{T} \cdot F_{k}$,

6.- update the log-conductivity: $\sigma^{m+1}:=\sigma^{m}+\delta$,

7.- if the stopping criterion is not met, set $m:=m+1$ and go to step 2 .

Algorithm 2: fine conductivity estimation (zero memory)

Same as algorithm 1 above except:

4.- compute $\mathrm{d} F_{k}^{T} \cdot F_{k}, k=1 \ldots, N$, using equation (31)

5.- solve for $\delta$ the equation $\left(\sum_{1 \leq k \leq N} \mathrm{~d} F_{k}^{T} \cdot \mathrm{d} F_{k}\right) \delta=-\sum_{1 \leq k \leq N} \mathrm{~d} F_{k}^{T} \cdot F_{k}$, using conjugate gradient,

4.2. Nested grids identification of the conductivity. The conductivity distribution is retrieved from $E_{1}\left(\sigma^{\star}\right), \ldots, E_{N}\left(\sigma^{\star}\right)$ by the following nested grids algorithm that is a combination of algorithms 1 and 2. We refer to this approach as a "nested grids" approach rather than a nested grid approach, even though multiple grids are used, in order to avoid a confusion with what is usually refered to as multigrid [7]. In particular, no use is made of the potentials on coarser grids to evaluate potentials on finer ones.

\section{Algorithm 3: combined algorithm}

input: the discretisation mesh, the values of the boundary currents $g_{1}, \ldots, g_{N}$ and the associated measurements $E_{1}\left(\sigma^{\star}\right), \ldots, E_{N}\left(\sigma^{\star}\right)$, initial guess for the log-conductivity

1.- set $n:=1$, set $\omega_{1}=\omega$, estimate the log-conductivity $\sigma$ provided by algorithm 1 .

2.- define a new partition of $\Omega$ by dividing each $\left(\omega_{i}\right)_{1 \leq i \leq n}$ in 2 subdomains (that are unions of convexes of the mesh), set $n:=2 n$,

3.- find an optimal log-conductivity $\sigma$ that is constant in each $\omega_{i}$ using algorithm 1 .

4.- if $n>20$, go to step 5 . else go to step 2 .

5.- the partition of $\Omega$ is the partition defined by the convexes of the mesh.

6.- find the log-conductivity $\sigma$ that is constant in each convex using algorithm 2 .

\section{Numerical Results using the COST funCtion $\mathcal{J}_{1}$}

In this section, we document the efficiency of the reconstruction method using $\mathcal{J}_{1}$. A steepest descent algorithm with adaptive step was implemented using FreeFem++ [8] for the minimisation of $\mathcal{J}_{1}$ (however we do not detail this gradient descent or steepest descent - algorithm). Several boundary conditions $g_{1}, \ldots, g_{N}$ are used to estimate the conductivity. The cost function is the sum of the cost-functions associated to these boundary conditions: $\mathcal{J}_{1}=\sum_{1 \leq k \leq N} \mathcal{J}_{1}^{k}$.

Note that in contrast with the theoretical reconstruction done in Section 2, we only make use of the diagonal data, that is, $S_{i i}, i=1, \ldots, N$, and we make no assumption on the regularity of these data. 
We study a test case that was introduced in [2]. On a disk of diameter 8, different conductivities are set, the background conductivity is equal to 0.5 , the conductivity in the small disk to 0.75 , the one in the triangle to 0.15 and finally to 2.55 in the L-shaped domain (see Figure 2). On the annulus of inner radius 6 and outer radius 8 , the conductivity is supposed known, equal to 0.5. Dirichlet boundary data are fixed to be equal to the Cartesian coordinates $g_{1}=x, g_{2}=y, g_{3}=x+y$ and $g_{4}=x-y$ on the boundary of the circle.

Note that $S_{33}=S_{11}+S_{22}+2 S_{12}$ and $S_{44}=S_{11}+S_{22}-2 S_{12}$, and the knowledge of this set of four data is actually equivalent to the knowledge of the hypothesis of the theoretical reconstruction done in Section 2.

5.1. The whole domain. The first reconstruction test is performed with $\omega=\Omega$, that is, $S_{i i}, i=1, \ldots, 4$ is known everywhere. The reconstuction experiments are conducted in three different cases. The first case is intended to document the convergence of the algorithm in a very favorable situation. The same mesh used to compute the reconstruction and to obtain the synthetic data. This mesh is a $50 \mathrm{~K}$ structured triangulation adapted to the shape of the inhomogeneities We then consider the same synthetic data, but no a priori information is contained in the reconstruction mesh. We use a uniform Delaunay mesh of the ball of $110 \mathrm{~K}$ triangles. A third test is performed with two different meshes, and a where the synthetic data is perturbed by a $10 \%$ multiplicative random noise. Finally, we tested the effect of sub-sampling, that is, when only partial averaged data is available.

Our initial guess for the conductivity inside the inner circle or radius 6 was 1 -any arbitrary number would do the same. Convergence curves are reported on Figure 3. For each of the three cases, we represent the evolution of the functional $\mathcal{J}_{1}$ to be minimised (on the left) and the $L^{1}$ error of $\sigma_{\text {comp }}$, the computed log-conductivity compared to $\sigma^{\star}$, the target log-conductivity (on the right). In practical applications, the correct conductivity is unknown: it is presented here to document the efficiency of the algorithm.

The test with a single mesh was performed to verify the convergence of the algorithm to the correct minimiser in an ideal situation. The evolution of the objective function and of the $L^{1}$ error towards machine precision leaves no doubt on the efficiency of the scheme. When the meshes are different, or when noise is added, the reconstruction is naturally less precise. On Figure 4 the absolute value of the relative differences between the computed and synthetic conductivity are plotted. The grey-scale used varies between 0 and $10 \%$.

In the noiseless cases (left), the plot clearly shows that the conductivity is recovered inside the shapes and on the background. The error is located only on the boundaries of the inclusions. This could be an effect of the mesh, since the boundaries cross the reconstruction mesh in a non conformal way. Figure 4 (left) also shows that shape corners create discrepancies. Around these points, the correct potential is singular whereas the reconstructed potential inherits additional smoothness from the uniform mesh.

When noise is added, Figure 4 (right) shows that a $10 \%$ multiplicative noise leads to a relative loss of precision on the conductivity of about $10 \%$, which is the best one can expect.

In Section 3.3, it was mentioned that to avoid loss of convexity in the descent, one could choose to pick, at each iteration, either the positive direction $\delta_{+}=\max (\delta, 0)$ or the negative one $\delta_{-}=\min (\delta, 0)$. We show the result on the Figure 3 , for one mesh, when $\delta_{ \pm}$is picked if its $L^{2}$ norm is the largest. The rate of convergence is similar to the one observed before. If instead $\delta_{+}\left(\right.$resp. $\left.\delta_{-}\right)$is used every other iteration, the convergence curve is more step-like, but the overall rate is the comparable. 

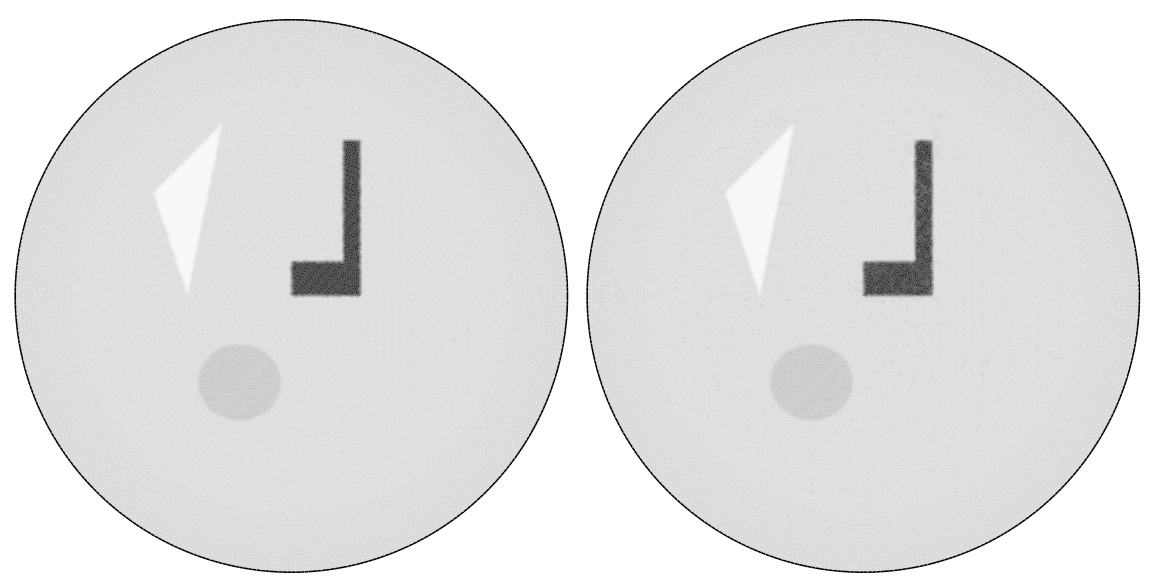

FiguRE 2. The reference material (left) and the reconstruction with $10 \%$ noise (right). The conductivities are 0.5 for the background, 0.15 for the triangle, 0.75 for the disc and 2.55 for the L-shaped region. The computations were done using FreeFem++, with $\mathrm{xd} 3 \mathrm{~d}$ for the rendering.
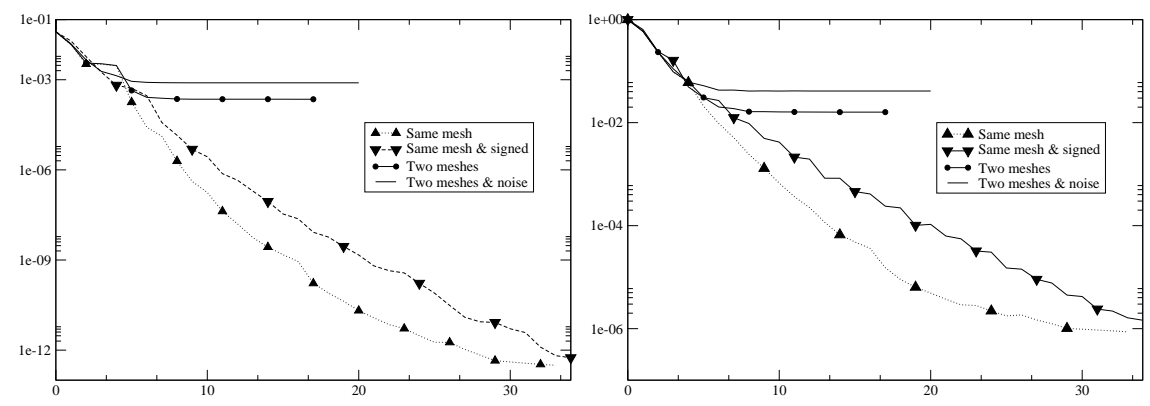

FiguRE 3. Convergence histories for the whole domain. The objective function $\mathcal{J}_{1}$ is on the left, and $L^{1}$ norm of the true error in conductivity is on the right. The dotted, triangle up, is for the one mesh noiseless case. The dashed, triangle down, curve corresponds to the case when only positive or negative increments are considered at each step. The solid line with disks corresponds to a reconstruction on a uniform mesh, with no added noise, and the plain solid line is for a uniform mesh and $10 \%$ noise.

Note that this reconstruction method is slower than the one in [2]. However, because it is based on a minimisation approach, it is more stable. Additionally, it requires only one current data-set, whereas the method presented in [2] cannot be used in that case.

We also tested the effect of interpolation error, in thet spirit of the examples presented in [2]. The data is first synthetised on a fine adapated mesh. It is then interpolated on a coarser uniform mesh. It is then reconstructed on a finer, but uniform, mesh. The coarser mesh is a $20 \mathrm{~K}$ triangles Delaunay mesh of a ball. This test amounts to average the data, it corresponds to the physical situation where the average of the density of energy on small sets is measured and not the point-wise energy. As it can be seen on Figure 5, the reconstruction is satisfying. It is to be compared with the coarse mesh interpolation of conductivity, displayed on its left. 


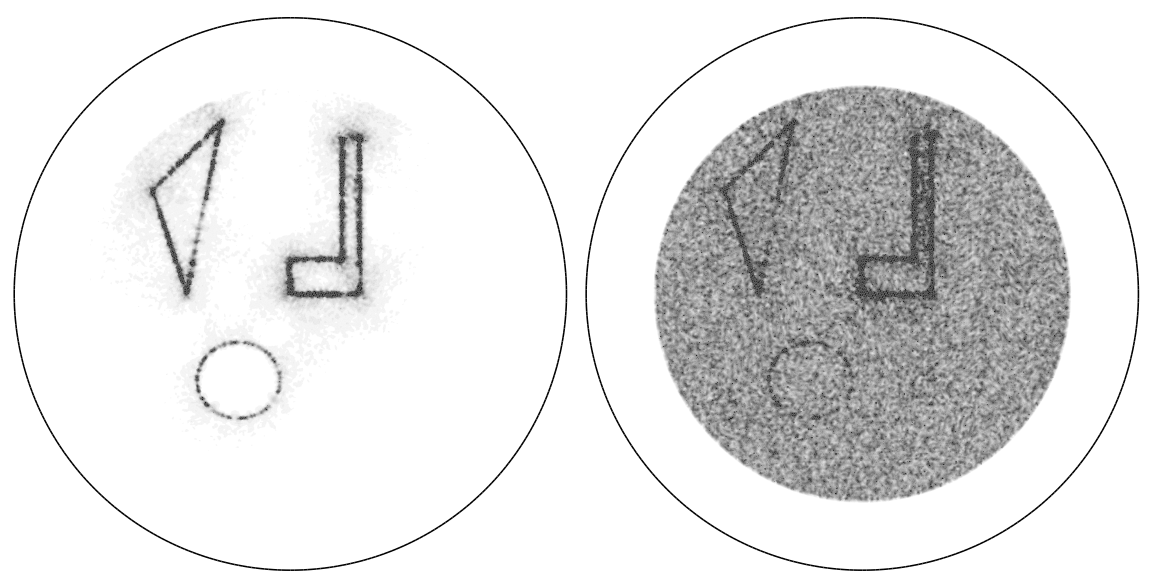

FiguRE 4. The absolute relative difference for the conductivity for the whole domain without noise (left) or with a $10 \%$ multiplicative noise (right). The white areas correspond to a $0 \%$ error and black one to $10 \%$ error or more.

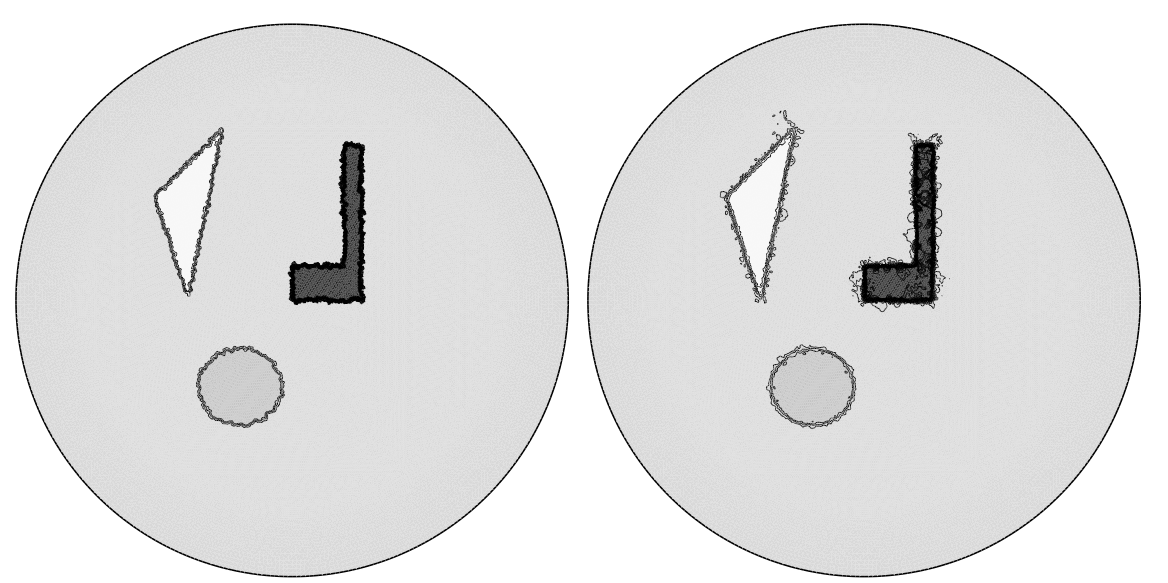

FiguRE 5. Interpolation of the data on a coarser mesh, the interpolation of the conductivity (left) and the computed conductivity (right).

5.2. Measurements in a smaller domain, and dependence on the outerdomain. We then tested the case when the energy density data was available only in an ellipse $\omega$ around the L-shaped domain. The reconstruction is shown in Figure 6 (left). Again, the resulting conductivity approaches well the reference one. This test validates the capacity of the algorithm to focus on smaller patches, hence having the ability to zoom on region of interest. The mesh used for this test is adapted to the ellipse: it is refined inside the ellipse, and uniform outside. An interesting feature of this test is the tendency of the algorithm to compensate discrepancies of the conductivity outside $\omega$ within a boundary layer in the neighbourhood of $\omega$. A close look at Figure 6 (left) shows that the higher conductivity on the left boundary of the ellipse stands for the triangle, and the one on the upper side is the upper branch of the L-shape that is not contained in the ellipse. Note that the computed conductivity is found only up to a multiplicative constant. This corroborates the study done in Section 2, for more regular conductivities. 


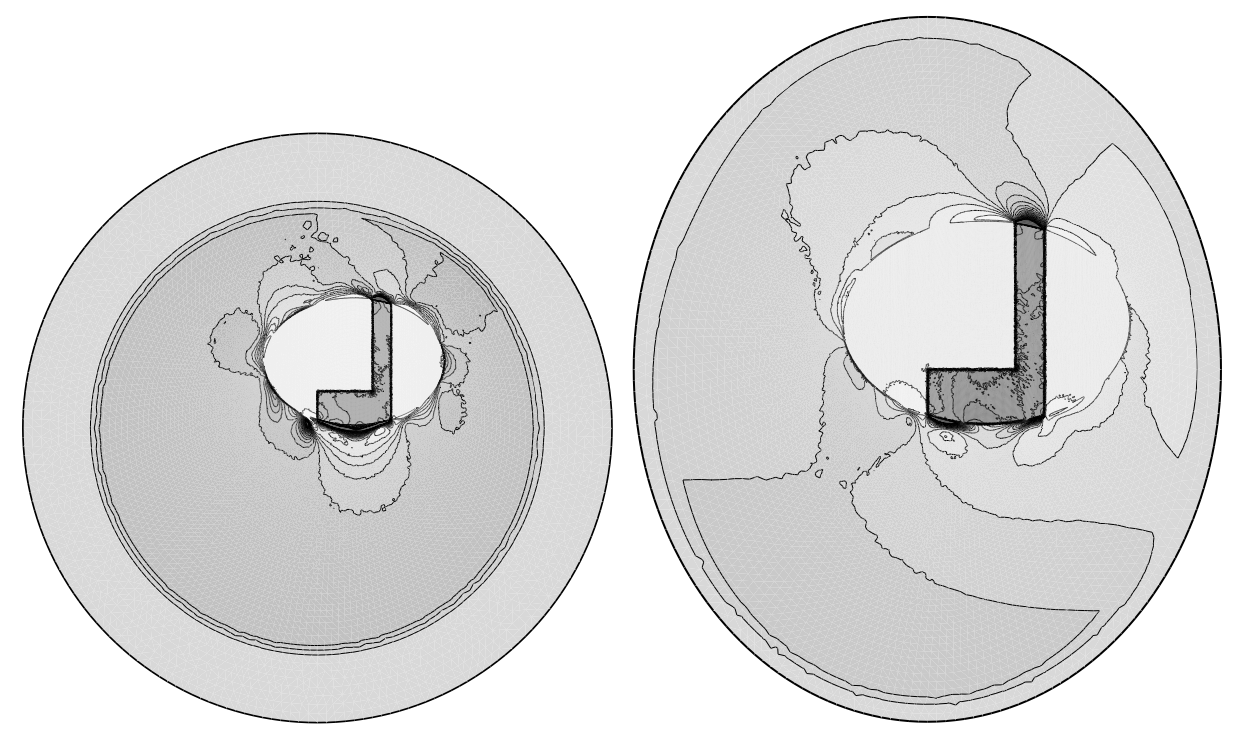

FiguRE 6. Reconstruction from partial data. On the left, the reconstruction inside an elliptic region, with a one mesh. One the right, the reconstruction when the domain outside the ellipse is changed, with the same data as on the left.

A natural question is whether such a reconstruction is still possible when the domain $\Omega$, and the Dirichlet boundary data $g_{i}$ is only approximately known. This is of practical importance, because for in vivo experiments, the exact geometry of the boundary, the human body, is not precisely known.

In this test, the elliptic measurement subdomain is preserved, but we attempt to recover $\sigma$ with a domain $\Omega$ that has been changed into a smaller ellipse. The Dirichlet boundary data imposed are the Cartesian coordinates data $g_{i}$ previously used, $x, y, x+y, x-y$, but they are now imposed on this new domain. Because they are not the $\mathrm{e}^{\sigma^{\star}}$-harmonic extensions of the previous boundary conditions, an error is introduced in the boundary conditions.

The resulting computed conductivity $\sigma_{\text {comp }}$ is given in Figure 6 (right).

The absolute relative error between the computed conductivity and the synthetic data inside the ellipse is shown in Figure 7, on a grey scale between 0 and $10 \%$. Note that since the conductivity is recovered up to a constant, the result was rescaled by a multiplicative factor (1.72) for the comparison. In this example, $89 \%$ of the area corresponds to less than $10 \%$ relative error.

5.3. Solving with a patchwork approach. The previous tests show that the conductivity can be recovered within a "patch", a sub-region of the domain. We now investigate whether the algorithm can be parallelised with multiple computers recovering different patches. Since no constraint is imposed on the conductivity outside the area of interest, Figure 6 shows that the minimisation procedure tends to create important errors outside the optimised patch $\omega$, in a boundary layer surrounding $\omega$. Furthermore, it reconstruct the conductivity up to a constant factor. which a priori depends of the sub-region. A natural concern is whether these error would render parallelisation inefficient.

The following numerical trial is an attempt to address this question. Given two overlapping patches and two adapted meshes, we compute separately the two steepest direction of descent, we interpolate the direction of descent on the other 


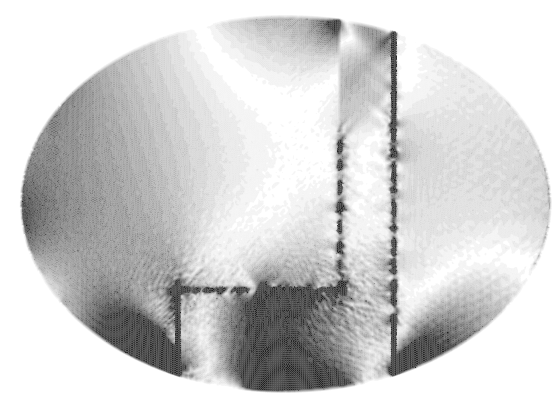

FiguRE 7. Absolute relative difference between the synthetic data and the reconstructed conductivity shown in Figure 6 (right). The reconstructed conductivity is rescaled by a 1.72 factor. The white areas correspond to $0 \%$ error and black one to $10 \%$ error or more.
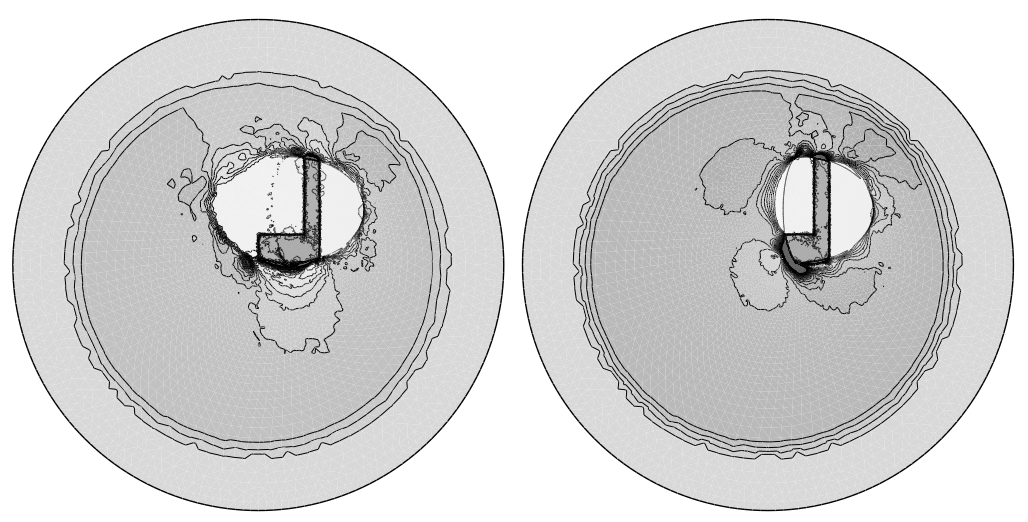

FIGURE 8 . The computed conductivity with the right patch only and then both patches.

mesh and average them. This averaged direction of descent is used to update the conductivity on each mesh.

The new objective function is just the sum of the objective functions for the patches. Figure 8 shows the resulting conductivities when the algorithm is applied with the right patch only and then when the algorithm take the two patches into consideration in a parallel algorithm.

Figure 9 shows the convergence histories. We compare the convergence of the cost function $\mathcal{J}_{1}$ when the optimisation is done on the complete ellipse with the patchwork approach. We see the rate of convergence comparable in all cases.

These convergence charts show that the parallel computation is as efficient as a global minimisation algorithm. Note that, since the meshes are refined on both patches, the mesh precision of the parallel algorithm is almost twice that of the global minimisation algorithm. This is the motivation of this parallelisation approach; to obtain a finer precision in a reduced time. The parallel algorithm is not less efficient than the optimisation on any of the two sub-regions. This shows that the error boundary layer which appear on the boundary of the patches is not a 


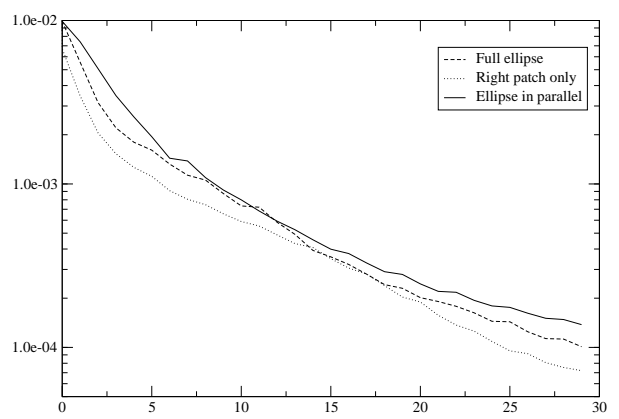

Figure 9. The convergence of the cost function $\mathcal{J}_{1}$ for a subregion. The dashed line corresponds to the full ellipse reconstruction shown in Figure 6 (left). The dotted line corresponds to the optimisation in the right patch only. The solid line corresponds to the two patches parallel optimisation.

severe drift from the optimal solution, as it vanishes when the outside domain is updated. The parallelisation of the algorithm is therefore relatively simple.

\section{Numerical Results using the COSt FunCtion $\mathcal{J}_{2}$}

In this section, the numerical results obtained for the cost function $\mathcal{J}_{2}$ are presented. Several boundary conditions $g_{1}, \ldots, g_{N}$ are used to estimate the conductivity. The cost function is the sum of the cost-functions associated to these boundary conditions: $\mathcal{J}_{2}=\sum_{1 \leq k \leq N} \mathcal{J}_{2}^{k}$. The algorithms were implemented with piecewise linear finite elements, using Getfem++ [10] and Matlab. The results presented below were obtained with the combined algorithm introduced in Section 4.2 (algorithm $3)$.

6.1. Two dimensional results: a half-disk. The domain $\Omega \subset \mathbb{R}^{2}$ is a half disk of radius 1 centered at the origin, the conductivity $\sigma$ is to be reconstructed in the half disk $\omega$ of radius 0.9 centered at the origin. The value of the conductivity smoothly varies between 1 and 1.8 in the background, and a circular inclusion was added, the radius of this inclusion is 0.06 and the contrast of the conductivity is 3 . For the generation of the data, the domain $\Omega$ is meshed by 6004 triangles and 3088 vertices, and the mesh is adapted to the geometry of the inclusion.

There are $N=2$ different boundary currents, respectively equal to $x_{1}$ and $x_{2}$ (the spatial coordinates) but for the bottom boundary of the domain that is considered as insulating (homogeneous Neumann condition). The local energy density is computed using piecewise affine finite elements. A gaussian noise is added, with variance equal to $1 \%$ of the $L^{2}$-mean of the energy. The energy density is then interpolated on a finer mesh with 16064 triangles and 8193 vertices, this mesh is independent of the geometry of the inclusion. This fine mesh is used for the reconstruction.

The subdomain $\omega$ contains 10400 convexes of the reconstruction mesh, this is the number of unknowns, and the number of data for each boundary current applied. The algorithm 3 was used with the following parameters : 6 nested grid steps, followed by 3 Gauss-Newton steps. In figure 10 the true conductivity (top left) and the estimated conductivity at several steps of algorithm are presented.

6.2. Three dimensional results: a half-sphere. A more realistic three dimensional geometry was simulated, in order to mimic breast examination. The domain $\Omega$ is a half-sphere of radius 1.1 , the domain $\omega$ is a half sphere of radius 0.9 . The 

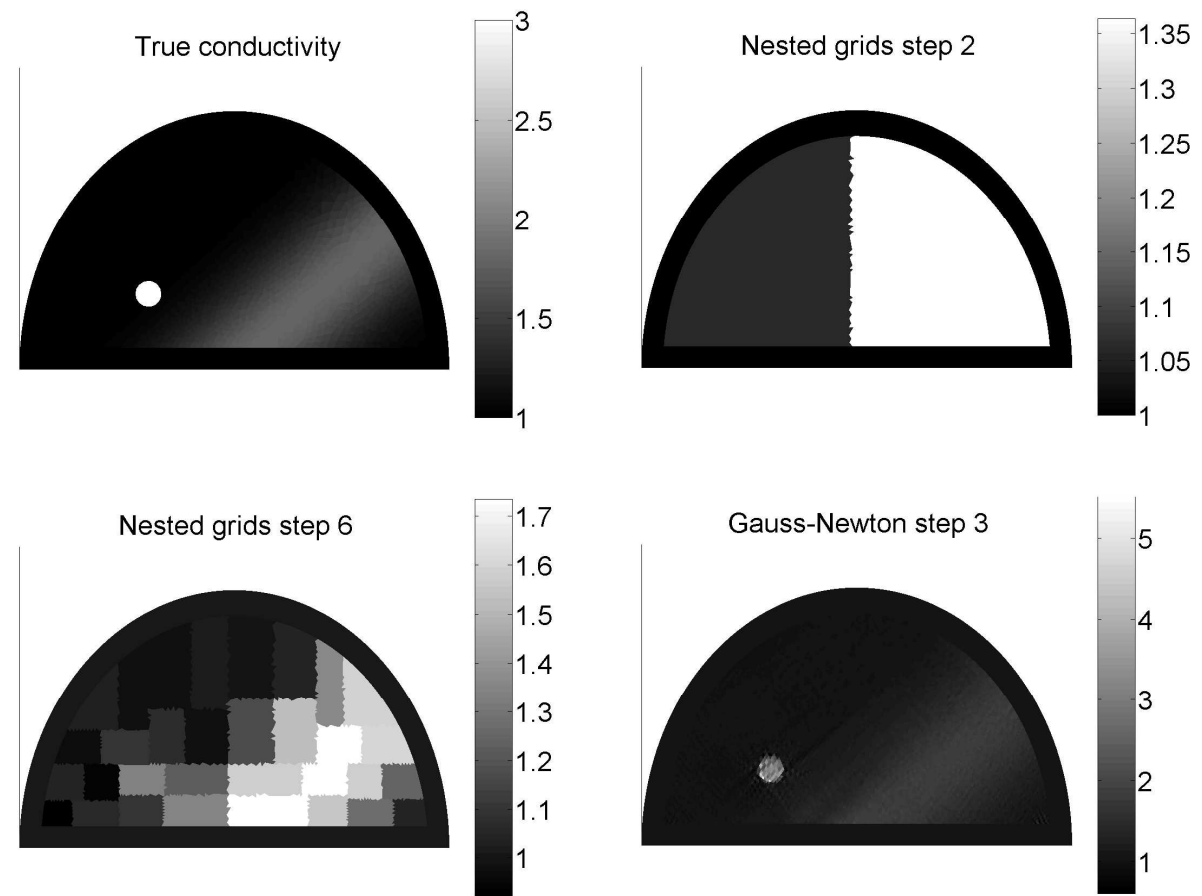

Figure 10. Reconstruction with $1 \%$ noise added. From left to right, first row: True conductivity ; nested grids step 2. Second row: nested grids step 6 ; Gauss-Newton step 3.

mesh of the domain $\omega$ contains 6321 convexes, this is the number of unknown conductivity coefficients. The numerical issues of interpolation between 3-dimensional meshes were not adressed in this work, and the reconstruction is performed on the same mesh as the mesh that was used to generate the data.

The bottom of the half sphere is insulating (homogeneous Neumann condition), and there are 8 disk-shaped electrodes at the boundary, see figure 11 . One experiment consists of applying a Dirichlet condition to the electrodes, such that each electrode is at a given electric potential. The true conductivity $\mathrm{e}^{\sigma^{\star}}$ is spacedependent, there is a spherical inclusion of radius 0.1 with high conductivity and a background where the conductivity depends smoothly on the second space variable. The quantity $\mathrm{e}^{\sigma^{\star}}\left|\nabla u^{\star}\right|^{2}$ is measured in $\omega$.

There are $N=4$ boundary conditions defined as follows: on each electrode a potential equal to respectively $g_{1}=x_{1}, g_{2}=x_{2}, g_{3}=x_{1}+x_{2}$ and $g_{4}=x_{1}-x_{2}$. where $x_{1}$ and $x_{2}$ are the first and second spatial coordinate of the centre of mass of the electrode. We show $g_{1}$ and $g_{2}$ on figure 12 .

The nested grid algorithm described in paragraph 4.2 was applied with the following parameters: two coarse nested grid steps and five fine Gauss-Newton steps. Figure 13 shows the logarithmic evolution of the discrepancy between the true conductivity and the reconstructed conductivity, as a function of the iterations (measured with different norms), with $0.01 \%$ and $2 \%$ Gaussian white noise. The complete resolution takes about $600 \mathrm{~s}$ with a $1.73 \mathrm{GHz}$ computer.

Note: other simulations were conducted with less boundary conditions $(N=$ 2 ). In the absence of noise, the results of the reconstruction were analogous to the reconstruction with $N=4$ different boundary conditions (but the speed is 


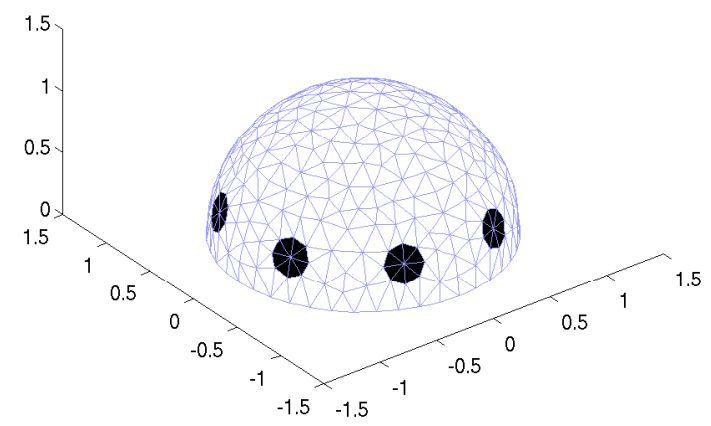

FiguRE 11. Location of the electrodes (black circles)
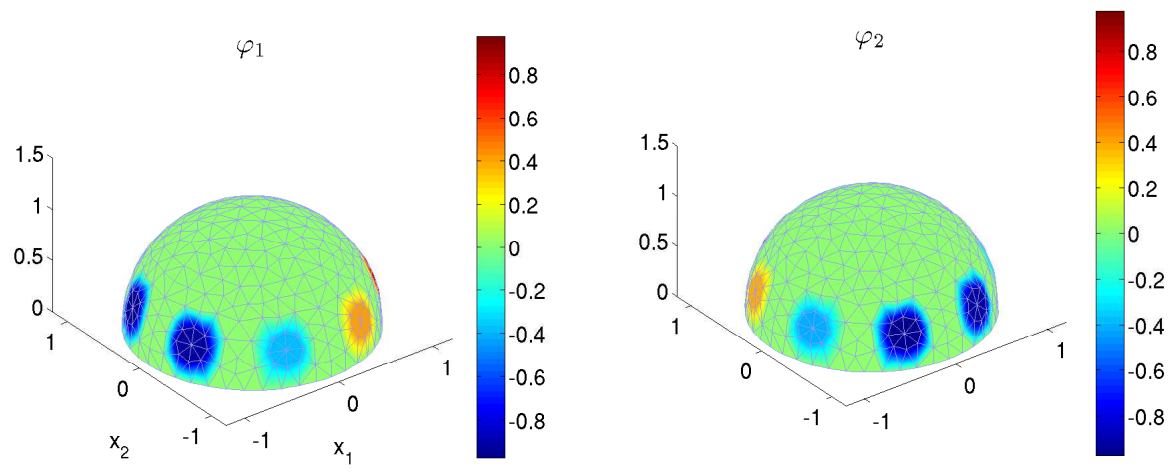

FiguRE 12 . The boundary conditions $g_{1}$ (left) and $g_{2}$ (right)

twice faster since the total number of conductivity problems to be solved is divided by a factor 2). However, in the presence of noise, taking into consideration 4 measurements allows to reduce the variance of the noise (and hence the error in the retrieved conductivity). An application to real data should evaluate the number of experiments that provides the best trade-off between speed and noise variance reduction.

\section{Conclusion}

The first result of this paper is to show that in two dimensions, under sufficient local regularity assumptions, the local energy densities corresponding to two diffeomorphic boundary data determine uniquely the conductivity up to a multiplicative constant. We were not able however to prove such a result in three dimensions, without prior information on the conductivity map. The second part of this paper illustrates that despite these limitations, the conductivity can be succesfully numerically reconstructed in two and three dimensions, even for irregular conductivity maps. We consider the reconstruction as a minimisation problem, and apply classical optimisation techniques. Although the Hessian of the functional we minimise fail to be strictly convex, choosing at each time-step either positive or negative 

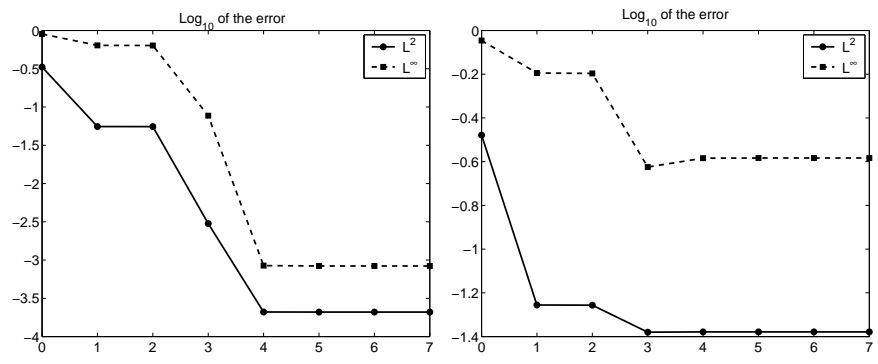

FiguRE 13. Evolution of the $\log _{10}$ of the discrepancy between the true and the reconstructed conductivity: $0.01 \%$ noise (left) and $2 \%$ noise (right)
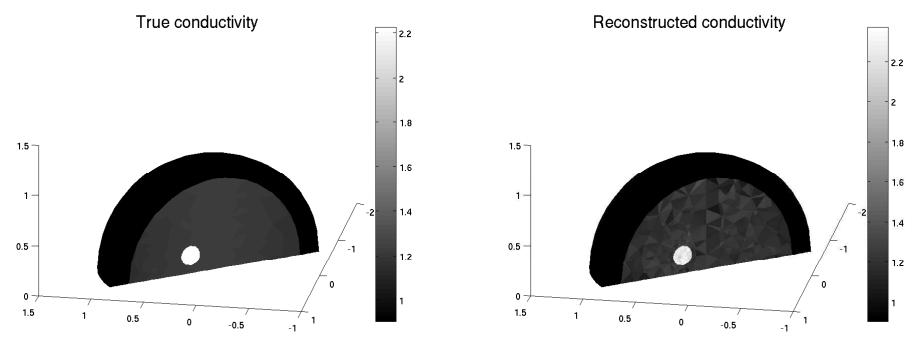

FIGURE 14. Slice views of the true and the reconstructed conductivity with $2 \%$ noise

increment allows to overcome that problem. We do not address the question of the theoretical well-posedness of the minimisation problem. Instead, we investigate it numerically. Due to computational power limitations, our numerical calculations in three dimensions are not exempt of some "inverse crime", namely, the same mesh is used for the creation of the synthetic data, and for the reconstruction. However, we did consider the case of noisy data. In two dimensions, however, we do not have such limitation and several computations are performed on independent meshes.

Our computations indicates that the problem is not very ill-posed in practice. The dependence on the boundary geometry is not severe, and in presence of $10 \%$ of white-noise, the error in the reconstructed conductivity map stays within approximately $10 \%$ of the target. This is a striking difference to the usual electrical impedance tomography problem, which is known to be very severely ill-conditioned (see e.g. [3]), and where numerical experiments such as the ones we conducted would have been fatal to the reconstruction. In particular, we do not need to rely on any Tikhonov regularisation, in contrast to what is done in [5] when only the Dirichlet to Neumann map is available.

Acknowledgement. This paper is the result of a collaboration which happened when all the authors were at the LMV (Laboratoire de Mathématiques de Versailles). This was made possible by the ANR project EchoScan (AN-06-Blan-0089). All the authors benefited from this grant, and gratefully acknowledge the support it provided.

This paper has been improved significantly by the suggestions of the referees, and we would like to thank them also. 


\section{REFERENCES}

[1] G. Alessandrini and V. Nesi. Univalent $\mathrm{e}^{\sigma}$-harmonic mappings. Arch. Rat. Mech. Anal., 158:155-171, 2001.

[2] H. Ammari, E. Bonnetier, Y. Capdeboscq, M. Tanter, and M. Fink. Electrical impedance tomography by elastic deformation. SIAM J. Appl. Math., 68(6):1557-1573, 2008.

[3] L. Borcea. Electrical impedance tomography. Inverse Problems, 18(6):R99-R136, 2002.

[4] M. Briane, G. W. Milton, and V. Nesi. Change of sign of the corrector's determinant for homogenization in three-dimensional conductivity. Arch. Ration. Mech. Anal., 173(1):133$150,2004$.

[5] D. C. Dobson. Convergence of a reconstruction method for the inverse conductivity problem. SIAM J. Appl. Math., 52(2):442-458, 1992.

[6] D. Gilbarg \& N.S. Trudinger. Elliptic Partial Differential Equations of Second Order. SpringerVerlag, Heidelberg 1983

[7] W. Hackbusch. Multigrid methods and applications, volume 4 of Springer Series in Computational Mathematics. Springer-Verlag, Berlin, 1985.

[8] F. Hecht, O. Pironneau, A. Le Hyaric, and K. Ohtsuka. FreeFem++. Laboratoire JacquesLouis Lions, UMR 7598, 2.20 edition, 2007.

[9] R. S. Laugesen. Injectivity can fail for higher-dimensional harmonic extensions. Complex Variables Theory Appl., 28(4):357-369, 1996.

[10] Y. Renard and J. Pommier. Getfem ++ , a generic Finite Element library in $C++$. Laboratoire MIP, UMR 5640, 2007.

MATHEMATICAL INSTITUTE, UNIVERSITY OF OXFORD, OXFORD OX1 3LB, UK E-mail address: YVES.CAPDEBOSCQ@MATHS.OX.AC.UK

IMT, UNIVERSITÉ PAUL SABATIER, CNRS, TOULOUSE, FRANCE

E-mail address: FEHREN@MIP.UPS-TLSE.FR

LMV, UNIVERSITÉ VERSAILLES-SAINT QUENTIN, CNRS, VERSAILLES, FRANCE E-mail address: FREDERIC.DE.GOURNAY@UVSQ.FR

LMV, UNIVERSITÉ VERSAILLES-SAINT QUENTIN, CNRS, VERSAILLES, FRANCE E-mail address: OTARED.KAVIAN@UVSQ.FR 